\title{
Fluctuation Analysis in Simple Fluids
}

\author{
A. Ziya Akcasu* \\ Cekmece Nuclear Research and Training Center, P.K.1, Hava Alani, Istanbul, Turkey \\ and \\ E. Daniels \\ University of Michigan, Ann Arbor, Michigan 48104
}

(Received 21 November 1969)

\begin{abstract}
A classical analysis of time correlations in simple fluids based on the generalized Langevin equation is presented. Formulas for the current-current correlations are developed explicitly in a region of frequencies $\left(\omega \sim 10^{13} \mathrm{sec}^{-1}\right)$ and wave numbers $\left(k \sim 10^{8} \mathrm{~cm}^{-1}\right)$ which are explored in typical slow-neutron-scattering measurements. Where applicable, comparisons are made with the results of the numerical calculations of Rahman in argonlike liquids, and good agreement is generally found. The analysis is based on a hydrodynamic description of fluids involving frequency- and wavelength-dependent transport parameters. The frequency and wavelength dependence of shear and longitudinal viscosities are given explicitly for argonlike liquids.
\end{abstract}

\section{INTRODUCTION}

The calculation of correlation functions in classical simple fluids in terms of microscopic quantities plays an important role in statistical mechanics, both for the interpretation of scattering experiments and the evaluation of the frequencyand wavelength-dependent transport coefficients. Among these, the density-density (or Van Hove) correlation function has received the most attention because of its direct relation to the differential scattering cross sections. Other correlation functions, such as the transverse current-current correlation functions, which are not readily accessible in experiments, have been subject to quantitative investigations only recently following the publication of Rahman's ${ }^{1}$ computer calculations in argonlike liquids. Computer studies of correlations ${ }^{2}$ using molecular dynamical calculations provide a stringent test of the validity of the various classical theories introduced in correlation analysis because they only assume a known model interparticle potential, and involve no quantum effects.

The classical analysis of correlations is usually based on either a kinetic or hydrodynamic description of fluids. The kinetic description developed extensively by Nelkin ${ }^{3}$ and his co-workers has been justified theoretically for dilute gases, ${ }^{4}$ and used successfully to interpret Brillouin scat tering from gases. ${ }^{5,6}$ It has also been extended to dense fluids ${ }^{7}$ and applied to Rahman's moleculardynamics calculations for liquid argon ${ }^{2}$ with poor quantitative agreement. $^{8}$

The hydrodynamic description of fluids has long been in use in the fluctuation analysis in arbitrary continuous media as a phenomenological theory.
In this approach, the conventional hydrodynamic equations are used to describe the linear response of the fluid, and the correlation functions are then related to the linear response by means of the fluctuation-dissipation theorem. ${ }^{9}$ Using the formulation described by Landau and Lifschitz, ${ }^{10}$ Rytov $^{11}$ applied the fluctuation-dissipation theorem to distributed parameter systems and calculated, among others, density-density correlation functions in an arbitrary continuous medium. This hydrodynamic approach has been used to interpret light scattering from liquids successfully by Mountain. ${ }^{12}$ A systematic and general hydrodynamic description of fluids for the calculation of correlations and transport coefficients has been developed by Kadonoff and Martin. ${ }^{13}$ The latter approach has been applied by Chung and Yip ${ }^{14}$ to Rahman's calculations of current-current correlations.

The objective of this paper is to present a classical analysis of correlations in simple fluids based on the generalized Langevin equation developed by Zwanzig ${ }^{15}$ and Mori ${ }^{16,17}$ and to interpret quantitatively the current-current correlations computed by Rahman ${ }^{2}$ for liquid argon. This approach has several appealing features. First by choosing the dynamical variable in the description of the fluid as the microscopic phase density function one obtains ${ }^{18}$ an exact kinetic equation for the correlation function $\Gamma\left(\overrightarrow{\mathrm{v}}, \overrightarrow{\mathrm{v}}^{\prime} ; \overrightarrow{\mathrm{x}}, \overrightarrow{\mathrm{x}}^{\prime}, t\right)$, which reduces by approximation to the kinetic equation derived by Zwanzig ${ }^{19}$ and Nelkin. ${ }^{8}$ However, by making an alternative choice of the dynamical variables to be microscopic densities in configuration space (e.g., mass, current, and energy densities) one arrives at an exact hydrodynamic 
description of correlation functions in terms of frequency- and wavelength-dependent transport parameters. The choice of the appropriate set of dynamical variables is arbitrary. For any choice of these variables one obtains exact expressions for the correlation functions of the variables in the set. Different levels of approximations can be obtained for a particular correlation function by adding new variables to the set and using the same simplifying assumption (e.g., Markov assumption) in each case. The continued fraction expansion of correlation functions by Mori, ${ }^{17}$ for example, can be obtained by using an orthogonal extension of the set starting from a given dynamical variable. The separation of thermodynamic and transport parameters can be given a geometric interpretation in terms of projections of dynamical variables on appropriate orthogonal axes, and the extension of their definitions to short wavelengths where the anisotropies become significant can be made in a systematic way.

In this paper, we will use the configurationspace (hydrodynamic) description of fluids to investigate the current-current correlation functions, and obtain approximate formulas for transverse and longitudinal current-current correlations in the frequency and wavelength regions encountered in neutron scattering using a Markov assumption. The results will then be compared to Rahman's computer data ${ }^{2}$ for liquid argon. We will also obtain the wavelength and frequency dependence of shear and longitudinal viscosities explicitly and discuss the influence of thermal effects as a function of wavelength. The objective of this paper is similar to that by Chung and Yip ${ }^{4}$; however their approach is based on the correlation-function formalism developed by Martin and Kadonoff, ${ }^{13}$ rather than on the projection-operator formalism by Zwanzig $^{15}$ and Mori. ${ }^{17}$

\section{GENERALIZED LANGEVIN EQUATION}

Extending the projection-operator technique first introduced by Zwanzig, ${ }^{15}$ Mori ${ }^{16}$ proves that the equation of motion of a set of dynamical variables $a_{j}(t)$ can be written in the form

$$
\frac{d \underline{a}(t)}{d t}-i \underline{\Omega} \cdot \underline{a}(t)+\int_{0}^{t} \underline{\varphi}(t-u) \cdot \underline{a}(u) d u=\underline{f}(t), \quad \begin{gathered}
t \geqslant 0.1 \\
(2.1)
\end{gathered} .
$$

The state vector $a(t)$ is defined such that it has no invariant contribution, e.g. ,

$$
\underline{a}(t)=\underline{A}(t)-\langle\underline{A}(t)\rangle,
$$

where $\langle\ldots\rangle$ denotes the thermal average of the vector $A(t)$. Equation (2.1) is the generalized form of the Langevin equation ${ }^{10,20}$ in the stochastic theory of Brownian motion. The random-force vector $\underline{f}(t)$ is given formally by

$$
\underline{f}(t)=e^{t(1-P) i L}(1-P) \underline{\dot{a}} \quad(\underline{\dot{a}}=i L \underline{a}),
$$

where $L$ is the classical Liouville operator and $P$ is a projection operator defined for any arbitrary phase function $G(t)$ by

$$
P G(t)=\left\langle G(t) \underline{a}^{\dagger}\right\rangle \cdot\left\langle\underline{a a^{\dagger}}\right\rangle^{-1} \cdot \underline{a} .
$$

Here $a^{\dagger}$ denotes the row vector which is the Hermitian conjugate of $a$. The $\left\langle a a^{\dagger}\right\rangle^{-1}$ is the inverse of the square matrix $\left[\left\langle a_{i} a_{j}^{\dagger}\right\rangle\right]$ which is the static correlation matrix. It should be noted that the evolution of the force vector $f(t)$ is determined by the special propagator $e^{t(1-P) \bar{i} L}$, whereas the evolution of the state vector $\underline{a}(t)$ is given by

$$
\underline{a}(t)=e^{t i L} \underline{a} \text {. }
$$

It is shown by Mori ${ }^{16}$ that

$$
\left\langle\underline{f}(t) \underline{a}^{\dagger}\right\rangle \equiv 0, \quad t \geqslant 0 .
$$

The square matrices $\varphi(t)$ (damping function) and $\underline{\Omega}$ (frequency matrix) are defined by

$$
\begin{aligned}
\underline{\varphi}(t) & =\left\langle\underline{f}(t) \underline{f}^{\dagger}(0)\right\rangle \cdot\left\langle\underline{a a^{\dagger}}\right\rangle^{-1}, \\
i \underline{\Omega} & =\left\langle\underline{\dot{a} a}{ }^{\dagger}\right\rangle \cdot\left\langle\underline{a a^{\dagger}}\right\rangle^{-1} .
\end{aligned}
$$

Multiplying (2.1) by $\underline{a}^{\dagger} \cdot\left\langle\underline{a a^{\dagger}}\right\rangle^{-1}$ from the right-hand side, taking the thermal average of the resulting equation, and using (2.6) yield

$\frac{d \underline{R}(t)}{d t}-\underline{i \Omega} \cdot \underline{R}(t)+\int_{0}^{t} \underline{\varphi}(t-u) \cdot \underline{R}(u) d u=0, t \geqslant 0$

where $\underline{R}(t)$, the normalized dynamic correlation matrix, is defined by

$$
\underline{R}(t)=\langle\underline{a}(t) \underline{a}\rangle \cdot\left\langle\underline{a} \underline{a}^{\dagger}\right\rangle^{-1} .
$$

The one-sided Fourier transform of $R(t)$ is obtained from (2.9) as

$$
\begin{aligned}
& \underline{R}(i \omega)=[i \omega-\underline{i \Omega}+\underline{\varphi}(i \omega)]^{-1} \\
& \underline{\varphi}(i \omega)=\lim _{\epsilon \rightarrow 0} \int_{0}^{\infty} e^{-\left(i \omega_{+} \epsilon\right) t} \underline{\varphi}(t) d t
\end{aligned}
$$$$
\text { where }
$$

The projection technique enables one to find a closed set of linear equations for the correlation matrix $\underline{R}(t)$ when the state variables $a_{j}(t)$ are chosen as fluctuations from thermal equilibrium. The theory, although formally exact, serves only to transform the calculation from the direct computation of $R(t)$ to the computation of $\Omega$ and $\varphi(t)$. However, the frequency matrix $\Omega$ is determined from static correlations which are generally much easier to compute than the time-dependent correlation functions, and we may use approximations to compute the damping matrix $\varphi(t)$. In particular, we will consider representations in which we can make a Markov approximation on $\underline{\varphi}(t)$, viz. , 


$$
\underline{W}=\lim _{\omega \rightarrow 0} \underline{\varphi}(i \omega)
$$

to approximate the transform of the correlation matrix $\underline{R}(i \omega)$ by

$$
\underline{R}(i \omega) \cong[i \omega-i \underline{\Omega}+\underline{W}]^{-1} .
$$

We shall follow this formal procedure to calculate the current-current correlation function and its transform by choosing the components of the state vector as the spatial Fourier transforms of the local densities of conserved variables.

\section{TRANSVERSE CURRENT CORRELATIONS}

We first calculate the cosine transform of the transverse current correlation function as a simple application of the generalized Langevin equation, and then compare it with Rahman's computations. For this purpose, we choose the components of the state vector as

$$
\begin{aligned}
& a_{1}=J_{1}(\overrightarrow{\mathrm{k}}), \\
& a_{2}=\Pi_{31}(\overrightarrow{\mathrm{k}}),
\end{aligned}
$$

where $\vec{J}(\overrightarrow{\mathrm{k}})$ and $\Pi(\overrightarrow{\mathrm{k}})$ are the mass current density and the stress tensor, respectively. They are defined by

$$
\begin{aligned}
& J_{j}(\overrightarrow{\mathrm{k}}) \equiv \sum_{\alpha=1}^{N} m v_{j}^{\alpha} \exp \left(i \overrightarrow{\mathrm{k}} \cdot \overrightarrow{\mathrm{x}}^{\alpha}\right), \\
& \Pi_{i j}(\overrightarrow{\mathrm{k}}) \equiv \sum_{\alpha=1}^{N}\left(m v_{i}^{\alpha} v_{j}^{\alpha}+\frac{1}{2} \sum_{\substack{\beta=1 \\
(\beta \neq \alpha)}}^{N} \frac{x_{i}^{\alpha \beta} x_{j}^{\alpha \beta}}{\left|\overrightarrow{\mathrm{x}}^{\alpha \beta}\right|^{2}} P^{\alpha \beta}(\overrightarrow{\mathrm{k}})\right) e^{i \overrightarrow{\mathrm{k}} \cdot \overrightarrow{\mathrm{x}}^{\alpha}}, \\
& \text { where } \quad \overrightarrow{\mathrm{x}}^{\alpha \beta}=\overrightarrow{\mathrm{x}}^{\alpha}-\overrightarrow{\mathrm{x}}^{\beta}, \\
& P^{\alpha \beta}(\overrightarrow{\mathrm{k}})=\left.R \frac{d V(R)}{d R} \frac{1-e^{-i \overrightarrow{\mathrm{k}} \cdot \overrightarrow{\mathrm{R}}}}{-i \overrightarrow{\mathrm{k}} \cdot \overrightarrow{\mathrm{R}}}\right|_{\overrightarrow{\mathrm{R}}=\overrightarrow{\mathrm{x}}} ^{\alpha \beta}
\end{aligned}
$$

In these definitions, $\overrightarrow{\mathrm{x}}^{\alpha}$ and $\overrightarrow{\mathrm{v}}^{\alpha}$ denote the position and velocity of the $\alpha$ th particle in the system, and the subscripts $i$ and $j$ refer to the Cartesian components in a coordinate system in which $\vec{k}$ is parallel to the $z$ axis. With this choice of variables the static correlation matrix is diagonal, viz.,

$$
\left\langle\underline{a a^{\dagger}}\right\rangle=\left[\begin{array}{cc}
\left\langle a_{1} a_{1}^{*}\right\rangle & 0 \\
0 & \left\langle a_{2} a_{2}^{*}\right\rangle
\end{array}\right]
$$

since the variables $a_{1}$ and $a_{2}$ are, respectively, odd and even functions of the particle velocities so that $\left\langle a_{2} a_{1}^{*}\right\rangle=0$. Furthermore, a direct evaluation of the diagonal terms using (3.2) yields

$$
\begin{aligned}
(\beta / V)\left\langle a_{1} a_{1}^{*}\right\rangle & =\rho_{0}, \\
(\beta / V)\left\langle a_{2} a_{2}^{*}\right\rangle & =C_{44}(k) \\
& =\left(\frac{n}{\beta}+n^{2} \int d^{3} R g(R) \frac{\partial^{2} V}{\partial x^{2}} \frac{(1-\cos k z)}{k^{2}}\right),
\end{aligned}
$$

where $\beta=\left(1 / k_{B} T\right)\left(k_{B}=\right.$ Boltzmann's constant $), \quad V$ is the volume of the system, and $\rho_{0}$ is the equilibrium density. Here $C_{44}(k)$ is one of the elastic moduli calculated by Schofield. ${ }^{21}$ The frequency matrix $\Omega$ is calculated using (3.4) and (3.5), and noting $\overline{\dot{a}}_{1}=i k a_{2}$ as follows:

$$
\underline{\Omega}=k\left[\begin{array}{cc}
0 & 1 \\
C_{44}(k) / \rho_{0} & 0
\end{array}\right] .
$$

Since $(1-P) \dot{a}_{1}=i k(1-P) a_{2}=0$, the random force component $f_{1}(t)$ is identically equal to zero and the damping matrix $\underline{\varphi}$ has only one nonzero element,

$$
\underline{\varphi}(t)=\left[\begin{array}{cc}
0 & 0 \\
0 & \varphi_{22}(t)
\end{array}\right],
$$

where

$$
\varphi_{22}(t) \equiv\left\langle(1-P) \dot{a}_{2}^{*} e^{t(1-P) i L}(1-P) \dot{a}_{2}\right\rangle\left\langle a_{2} a_{2}^{*}\right\rangle^{-1} .
$$

The generalized Langevin equation for the set $\left[a_{1}(t), a_{2}(t)\right]$ becomes

$$
\begin{aligned}
& \frac{d a_{1}(t)}{d t}-i k a_{2}(t)=0 \\
& \frac{d a_{2}(t)}{d t}-i k \frac{C_{44}}{\rho_{0}} a_{1}(t)+\int_{0}^{t} \varphi_{22}(t-u) a_{2}(u) d u=f_{2}(t) .
\end{aligned}
$$

The transverse current correlation function is defined by

$$
R_{T}(k, t)=\frac{\left\langle a_{1}(t) a_{1}^{*}\right\rangle}{\left\langle a_{1} a_{1}^{*}\right\rangle} .
$$

Its cosine transform $R_{T}(k, \omega)$ is the transverse current power spectral density. The latter can be obtained directly from (3.8) by multiplying it by $a_{1}^{*}$, taking the thermal averages, and using $\left\langle f_{2}(t) a_{1}^{*}\right\rangle \equiv 0$ as

$$
R_{T}(k, \omega)=\operatorname{Re}\left[i \omega+\left(k^{2} / \rho_{0}\right) \eta_{T}(k, i \omega)\right]^{-1},
$$

where $\eta_{T}(k, i \omega)$, which we refer to as the transverse viscosity, ${ }^{22}$ is defined by

$$
\begin{aligned}
& \eta_{T}(k, i \omega) \equiv\left(\rho_{0} / i k\right)\left\langle a_{2}(i \omega) a_{1}^{*}\right\rangle /\left\langle a_{1}(i \omega) a_{1}^{*}\right\rangle, \\
& \eta_{T}(k, i \omega)=C_{44}(k) /\left[i \omega+\varphi_{22}(k, i \omega)\right] .
\end{aligned}
$$

Rahman $^{2}$ has computed $R_{T}(k, \omega)$ for various values $k$ and $\omega$. We have been able to obtain an exact expression for it in terms of the Laplace transform of the damping function, viz., $\varphi_{22}(k, i \omega)$. However, the evaluation of the latter using $(3.7 \mathrm{~b})$ is as difficult as solving the Liouville equation, although perturbative techniques such as expansions in density or interparticle potential ${ }^{18}$ may be used in dilute systems. For sufficiently small frequencies, we can approximate (3.10) by replacing $\varphi_{22}(k, i \omega)$ by its zero-frequency limit $\varphi_{22}(k, i \omega=0)$. This approximation corresponds to a Markov description of the fluid in terms of $a_{1}(t)$ and $a_{2}(t)$, in 
which the conivolution integral in $(3.8 \mathrm{~b})$ is replaced by

$$
\int_{0}^{t} d u \varphi_{22}(t-u) a_{2}(u) \cong a_{2}(t) \int_{0}^{\infty} d u \varphi_{22}(u) .
$$

The frequency range in which the Markov assumption may be expected to be valid can be estimated by considering the next term in the expansion of $\varphi_{22}(k, i \omega)$ in powers of $(i \omega)$ :

$$
\omega \ll\left|\varphi_{22}(k, i \omega)\left(\frac{d \varphi_{22}(k, i \omega)}{d(i \omega)}\right)^{-1}\right|_{i \omega=0} .
$$

It is clear that the Markov assumption ceases to be valid if $\varphi_{22}(k, i \omega=0)$, the leading term in this expansion, vanishes for some values of $k$. In such cases it turns out to be more convenient to go to a more complete description of the fluid by introducing new variables.

With these remarks, we obtain the following approximate form for $R_{T}(k, \omega)$ :

$$
R_{T}(k, \omega) \simeq \frac{\omega_{T}(k) k^{2} C_{44}(k) / \rho_{0}}{\omega^{2} \omega_{T}^{2}(k)+\left[k^{2} C_{44}(k) / \rho_{0}-\omega^{2}\right]^{2}},
$$

where we have introduced

$$
\omega_{T}(k)=\lim _{\omega \rightarrow 0} \varphi_{22}(k, i \omega) .
$$

Calculation of $\omega_{T}(k)$ directly from (3.14) is still a formidable task in dense fluids, although it is simpler than calculating $\varphi_{22}(k, i \omega)$. Therefore, we choose to try to guess its $k$ dependence by considering the asymptotic behavior of $\eta_{T}(k, i \omega)$ in the small- $k$ limit, and of $R_{T}(k, \omega)$ in the large- $k$ limit. We shall show in Sec. IV [cf. (4.54)] that

$$
\lim _{k, \omega \rightarrow 0} \eta_{T}(k, i \omega)=C_{44}(0) / \omega_{T}(0)
$$

is equal to the conventional shear viscosity $\eta_{s}$. Hence, the small- $k$ limit of $\omega_{T}(k)$ is given by

$$
\omega_{T}(0)=C_{44}(0) / \eta_{S}
$$

[note that $C_{44}(0)$ is $G_{\infty}$ in Zwanzig's notation ${ }^{22}$ ].

The large- $k$ behavior of $R_{T}(k, \omega)$ may be predicted from the transverse current spectrum of an ideal gas, viz. ,

$$
R_{T}^{I G}(k, \omega)=\left(\pi \beta m / 2 k^{2}\right)^{1 / 2} e^{-\beta m \omega^{2} / 2 k^{2}} .
$$

This function has a single maximum at $\omega=0$ for all $k$. On the other hand, (3.13) attains its maximum for a fixed $k$ at a frequency

$$
\omega_{T m}^{2}(k)=k^{2} C_{44}(k) / \rho_{0}-\frac{1}{2} \omega_{T}^{2}(k)
$$

for $\omega_{T}^{2}(k)<2 k^{2} C_{44}(k) / \rho_{0}$, and at $\omega=0$ otherwise. [ The function $\omega_{T m}(k)$ is of ten referred to as the dispersion relation. ${ }^{2}$ ] The dispersion relation (3.18) is expected to approach that of an ideal gas as $k \rightarrow \infty$ because for large $k$ the particles behave as free particles. (This is more apparent in the case of longitudinal current correlation function because it is related directly to the neutron scattering cross section where the large values of $k$ correspond to large momentum transfer to the scattering medium.) Hence, we require $\omega_{T m}^{2}(k)$ to approach zero for large values of $k$, i. e. ,

$$
\omega_{T}^{2}(k) \rightarrow 2 k^{2} C_{44}(k) / \rho_{0} .
$$

Thus, we obtain the asymptotic behavior of $\omega_{T}(k)$ for small and large $k$ from (3.16) and (3.19). The $k$ dependence of $\omega_{T}(k)$ for the intermediate values of $k$ can be obtained by interpolating it between the zero and large- $k$ limits by the following formula:

$$
\begin{aligned}
\omega_{T}^{2}(k)= & 2 k^{2} C_{44}(k) / \rho_{0} \\
& +\frac{\omega_{T}^{2}(0)-2 k^{2}\left(C_{44}(k)-\rho_{0} / \beta m\right) / \rho_{0}}{1+k^{2} / k_{0}^{2}},
\end{aligned}
$$

where $k_{0}$ is an adjustable parameter whose choice will be discussed presently.

It is interesting to compare (3.13) using the above expression for $\omega_{T}(k)$ to the ideal-gas result in (3.17) for zero frequency and large $k$, because the Markov approximation becomes exact at $\omega=0$. Noting that $C_{44}(k) / \rho_{0} \rightarrow(1 / \beta m)$ [cf. Eq. (3.5)], we obtain $R_{T}(k, 0)$ from $(3.13)$ as $(2 \beta m)^{1 / 2} / k$ whereas $(3.17)$ yields $(\pi \beta m / 2)^{1 / 2} / k$. The ratio is $(4 / \pi)^{1 / 2}$ $\approx 1.12$. Thus (3.13) recaptures the ideal-gas result in the limit of small $\omega$ and large $k$. It may be pointed out here that (3.10) can be approximated for large frequencies by replacing $\varphi_{22}(k, i \omega)$ by $\varphi_{22}(k, t=0) / i \omega$ [short-time expansion of $\left.\varphi_{22}(k, t)\right]$. Since our interest lies in the small-frequency region we shall not dwell on this point further even though $\varphi_{22}(k, t=0)$ is calculable exactly.

With the aid of (3.20) we have been able to obtain an expression for the transverse current power spectral density (3.13), which contains only one adjustable parameter $k_{0}$. The value of $k_{0}$ determines the transition from the small- to large$k$ limits. It is expected to be in the vicinity of the main peak of the structure function $S(k)$, which occurs at $k=2 \AA^{-1}$ in Rahman's computer calculations ${ }^{2}$ for liquid argon. We have chosen $k_{0}=1.5 \AA^{-1}$ which yielded a good fit to the computed curves although the value of $k_{0}$ is found to be not too critical. The other constant in $(3.20)$ is $\omega_{T}^{2}(0)$ which is obtained from (3.15) as $\omega_{T}^{2}(0)=0.1 \times 10^{26} \mathrm{sec}^{-2}$, corresponding to a value for the shear viscosity $\eta_{S}=2.8 \times 10^{-3} \mathrm{P}$ at $\rho_{0}=1.407 \mathrm{~g} / \mathrm{cm}^{3}$ and $T=76^{\circ} \mathrm{K}$ for liquid argon. The values of $C_{44}(k)$ were computed according to Eq. (3.5) in which the interparticle potential is taken, following Rahman, as

$$
V(R)=4 \epsilon\left[(\sigma / R)^{12}-(\sigma / R)^{6}\right]
$$

with $\left(\epsilon / k_{B}\right)=120^{\circ} \mathrm{K}\left(k_{B}=\right.$ Boltzmann constant $)$ and $\sigma=3.4 \AA$. The variation of $C_{44}(k)$ is plotted in Fig. 1. 


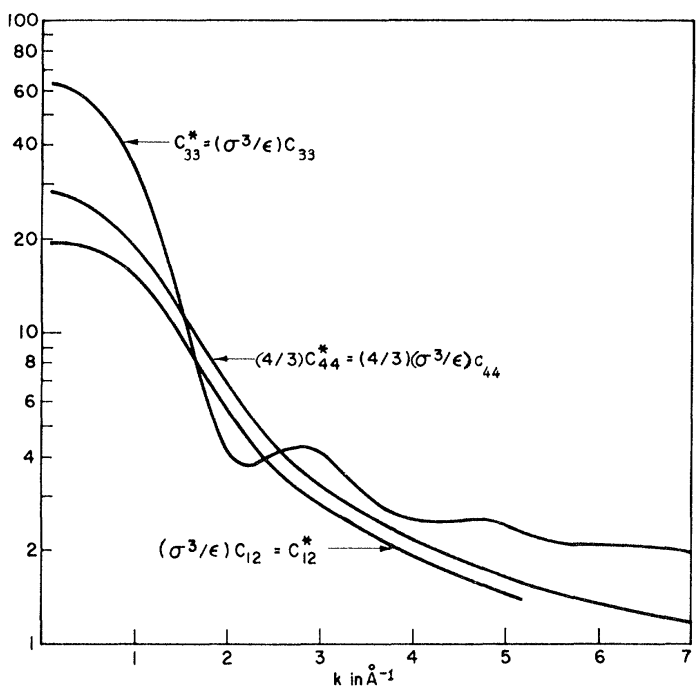

FIG. 1. Variation of elastic moduli with wavelength.

Figures 2 and 3 show the variation of $\omega_{T}(k)$ and $\eta_{T}(k, 0)=C_{44}(k) / \omega_{T}(k)$ with $k$. We observe that the $k$-dependent shear viscosity decreases very rapidly by a factor of 100 in the region of $k$ from zero to $2 \AA^{-1}$, and approaches zero as $1 / k$. This $k$ dependence of $\eta_{T}(k, 0)$ appears to be crucial to the behavior of $R_{T}(k, \omega)$ for the $k$ values in $1-4$ $\AA^{-1}$.

Figures 4 and 5 show comparison between the calculated curves and Rahman's data. ${ }^{2}$ It is noteworthy that the present model predicts well the cutoff wavelength in the dispersion curve, i. e., $\omega_{T m}(k)$. Other features are self-explanatory.

\section{GENERALIZED HYDRODYNAMIC DESCRIPTION}

The previous application indicates that the correlation function associated with a dynamical vari-

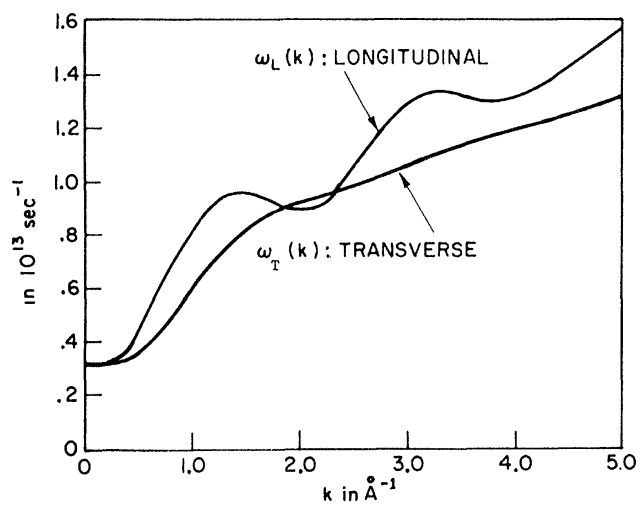

FIG. 2. Variation of the transverse and longitudinal relaxation frequencies with wavelengths.

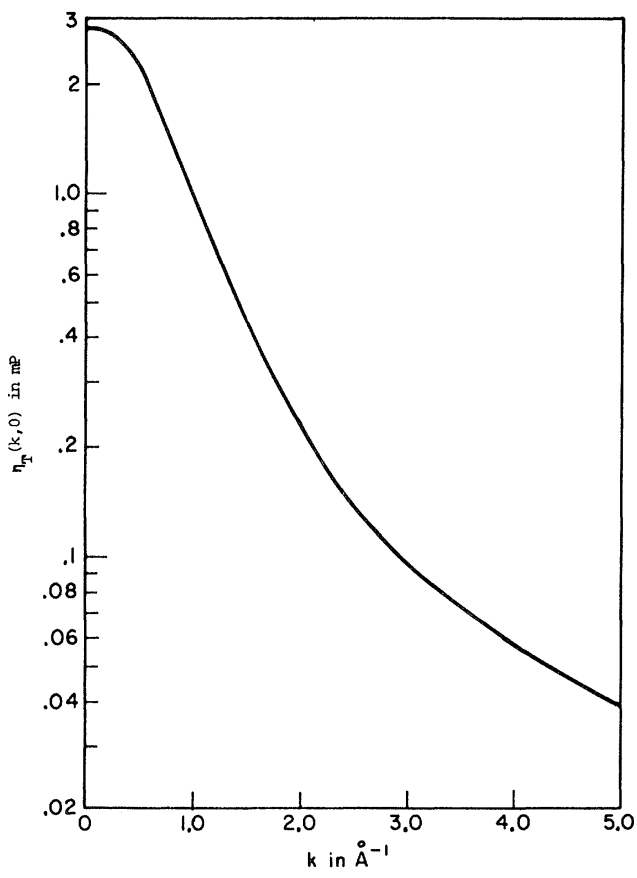

FIG. 3。Variation of shear viscosity with wavelength。

able $a_{1}(t)\left[\right.$ e.g., $\left.J_{1}(\overrightarrow{\mathrm{k}}, t)\right]$ can be obtained by solving the appropriate generalized Langevin equation. If only the autocorrelation function is of interest, the one-component description of the system is sufficient in principle. The correlation function in this case is obtained by solving

$$
\dot{R}_{1}(t)+\int_{0}^{t} d u \varphi_{1}(t-u) R_{1}(u)=0
$$

(note that the frequency matrix is always zero in one-dimensional description). The damping function $\varphi_{1}(t)$ involves $f(t)=\exp [t(1-P) i L](1-P) \dot{a}_{1}(0)$, where $P$ projects a phase function onto $a_{1}(0)$. Although (4.1) is exact, the calculation of $\varphi_{1}(t)$ is as difficult as calculating $\left\langle a_{1}(t) a_{1}^{*}\right\rangle$ directly. Crude approximations for $\varphi_{1}$, such as the Markov assumption, are generaily not precise enough to include even the qualitative features of the correlation function, or the power spectral density associated with it, for large values of $\omega$ and $k$. By introducing instead a multidimensional description of the system, one actually extracts a great deal of information about the collective motion of the system through the frequency matrix even though one may still be interested only in the autocorrelation function of a single variable. This information is contained in $\varphi_{1}(t)$ in one-dimensional description. A proper choice of the additional variables in a given system can lead to a sufficiently precise expression for the correlation function in a wide range of $\omega, k$ even with crude approximations 

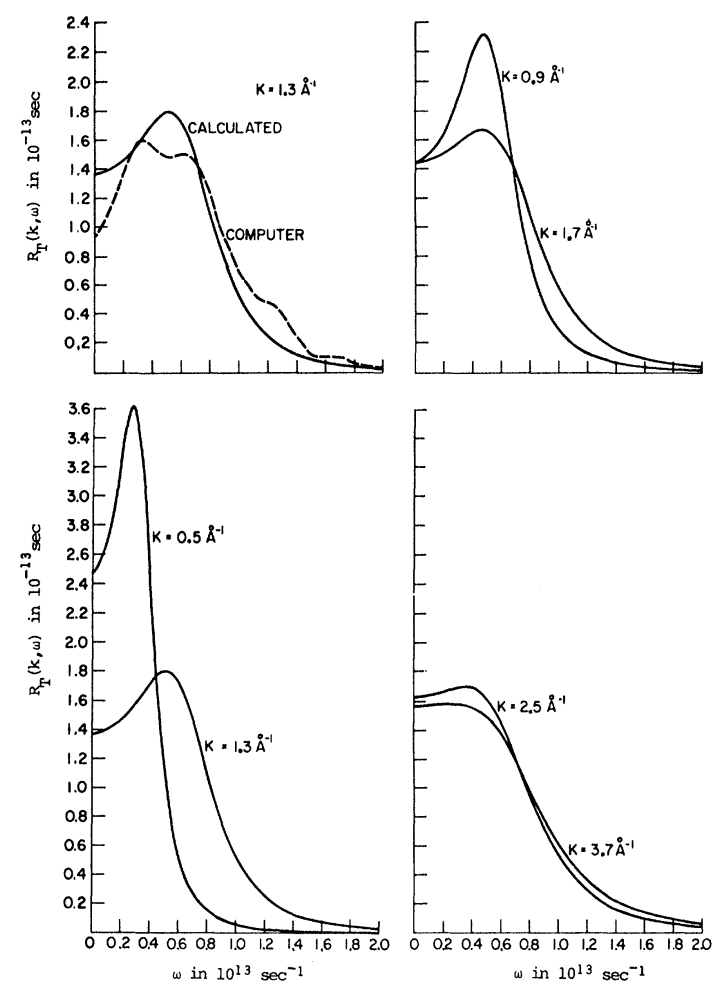

FIG. 4. Transverse current-current correlation $R_{\perp}(k, \omega)$ versus $\omega$ for various $k$.

on the multidimensional damping function. The variables $J_{1}(\overrightarrow{\mathrm{k}})$ and $\Pi_{31}(\overrightarrow{\mathrm{k}})$ introduced in Sec。III provide such a description for the transverse current correlations. The description of the longitudinal current correlations requires a more detailed description of the fluid including thermal and viscosity effects as will be demonstrated in this section. Moreover, a multidimensional description also allows the computation of the various cross-correlations between the variables in the set in terms of the same thermodynamic and transport parameters.

The purpose of this section is then to present a 14-dimensional description of a simple liquid, and to compute specifically the transverse and longitudinal current correlations. This description includes thermal effects, and sheds light on the anisotropies in the fluid for large- $k$ values.

For state variables, choose

$$
\underline{\mathrm{a}} \equiv \operatorname{col}\left[\rho, \theta, \sigma_{\mu}, J_{i}, q_{j}\right] \text {, }
$$

where $J_{i}$ and $q_{j}$ are vectors with three components $(i, j=1,2,3)$ and $\sigma_{\mu}$ is a six-component vector with $\mu=1, \ldots, 6$, ) viz. ,

$$
\sigma_{\mu}=\operatorname{col}\left[\sigma_{11}, \sigma_{22}, \sigma_{33}, \sigma_{13}, \sigma_{23}, \sigma_{12}\right] .
$$

The variables $\theta, \sigma_{\mu}$, and $q_{j}$ are defined by

$$
\begin{aligned}
& \rho(\overrightarrow{\mathrm{k}})=m \sum_{\alpha=1}^{N} e^{i \overrightarrow{\mathrm{k}} \cdot \overrightarrow{\mathrm{x}}^{\alpha}}, \\
& \theta(\overrightarrow{\mathrm{k}})=\left(E(\overrightarrow{\mathrm{k}})-\frac{\left\langle E(\overrightarrow{\mathrm{k}}) \rho^{*}(\overrightarrow{\mathrm{k}})\right\rangle}{\left\langle\rho(\overrightarrow{\mathrm{k}}) \rho^{*}(\overrightarrow{\mathrm{k}})\right\rangle} \rho(\overrightarrow{\mathrm{k}})\right), \\
& \sigma_{i j}(\overrightarrow{\mathrm{k}}) \equiv \Pi_{i j}(\overrightarrow{\mathrm{k}})-\frac{\left\langle\Pi_{i j}(\overrightarrow{\mathrm{k}}) \rho^{*}(\overrightarrow{\mathrm{k}})\right\rangle}{\left\langle\rho(\overrightarrow{\mathrm{k}}) \rho^{*}(\overrightarrow{\mathrm{k}})\right\rangle} \rho(\overrightarrow{\mathrm{k}}) \\
& \\
&-\frac{\left\langle\Pi_{i j}(\overrightarrow{\mathrm{k}}) \theta^{*}(\overrightarrow{\mathrm{k}})\right\rangle}{\left\langle\theta(\overrightarrow{\mathrm{k}}) \theta^{*}(\overrightarrow{\mathrm{k}})\right\rangle} \theta(\overrightarrow{\mathrm{k}}), \\
& q_{j}(\overrightarrow{\mathrm{k}}) \equiv Q_{j}(\overrightarrow{\mathrm{k}})-\left\langle Q_{j} \overrightarrow{\mathrm{J}} *(\overrightarrow{\mathrm{k}})\right\rangle \cdot\langle\overrightarrow{\mathrm{J}}(\overrightarrow{\mathrm{k}}) \overrightarrow{\mathrm{J}} *(\overrightarrow{\mathrm{k}})\rangle^{-1} \cdot \overrightarrow{\mathrm{J}}(\overrightarrow{\mathrm{k}}) .
\end{aligned}
$$

The definitions of $\overrightarrow{\mathrm{J}}(\overrightarrow{\mathrm{k}})$ and $\Pi_{i j}(\overrightarrow{\mathrm{k}})$ have already been given in (3.2a) and (3.2b), respectively. The quantities $E(\overrightarrow{\mathrm{k}})$ and $Q_{j}(\overrightarrow{\mathrm{k}})$ are the energy density and the energy current density, respectively. They are defined as
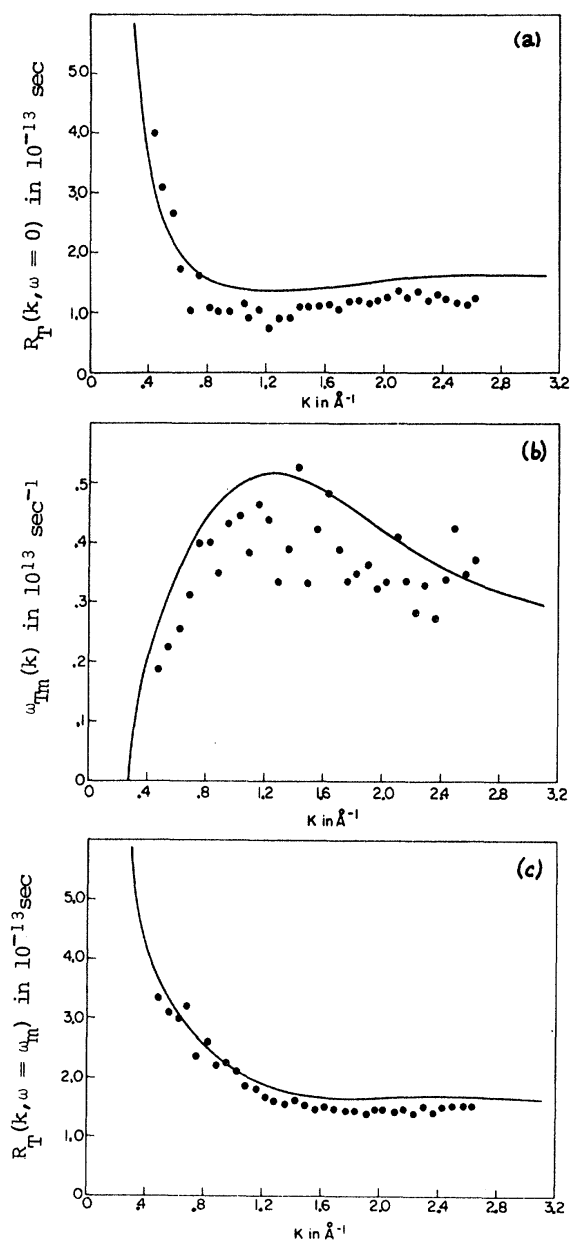

FIG. 5. (a) Maxima of a transverse current-current correlation function versus wave number $k$. Solid line due to present theory and points represent 'Rahman's data. (b) Frequency at which the transverse currentcurrent correlation function is maximum. (c) Transverse current-current correlation at zero frequency. 


$$
\begin{aligned}
E(\overrightarrow{\mathrm{k}})= & \sum_{\alpha=1}^{N}\left(\frac{1}{2} m \overrightarrow{\mathrm{v}}^{\alpha} \cdot \overrightarrow{\mathrm{v}}^{\alpha}+\frac{1}{2} \sum_{\substack{\beta=1 \\
(\beta \neq \alpha)}}^{N} V\left(\left|\overrightarrow{\mathrm{x}}^{\alpha}-\overrightarrow{\mathrm{x}}^{\beta}\right|\right)\right) e^{i \overrightarrow{\mathrm{k}} \cdot \overrightarrow{\mathrm{x}}^{\alpha}}, \\
Q_{\mathrm{j}}(\overrightarrow{\mathrm{k}})= & \sum_{\alpha} v_{j}^{\alpha}\left(\frac{1}{2} m \overrightarrow{\mathrm{v}}^{\alpha} \cdot \overrightarrow{\mathrm{v}}^{\alpha}+\frac{1}{2} \sum_{\substack{\beta=1 \\
(\beta \neq \alpha)}}^{N} V\left(\left|\overrightarrow{\mathrm{x}}^{\alpha}-\vec{x}^{\beta}\right|\right)\right) e^{i \overrightarrow{\mathrm{k}} \cdot \overrightarrow{\mathrm{x}}^{\alpha}} \\
& +\frac{1}{4} \sum_{\alpha, \beta}^{\prime \prime}\left(\overrightarrow{\mathrm{v}}^{\alpha}+\overrightarrow{\mathrm{v}}^{\beta}\right) \cdot \overrightarrow{\mathrm{x}}^{\alpha \beta} \frac{x_{j}^{\alpha \beta}}{\left|\overrightarrow{\mathrm{x}}^{\alpha \beta}\right|^{2}} P^{\alpha \beta}(\overrightarrow{\mathrm{k}}) e^{i \overrightarrow{\mathrm{k}} \cdot \overrightarrow{\mathrm{x}}^{\alpha}}
\end{aligned}
$$

$\left[\overrightarrow{\mathrm{x}}^{\alpha \beta}\right.$ and $P^{\alpha \beta}(\overrightarrow{\mathrm{k}})$ were defined previously in $(3.2 \mathrm{c})$ and $(3.2 \mathrm{~d})]$.

The following usual conservation laws prevail among the variables $\rho, J_{j}, \Pi_{i j}, E$, and $Q_{j}$ : (a) $\frac{\partial \rho}{\partial t}=i \overrightarrow{\mathrm{k}} \cdot \overrightarrow{\mathrm{J}}, \quad$ (b) $\frac{\partial \overrightarrow{\mathrm{J}}}{\partial t}=i \overrightarrow{\mathrm{k}} \cdot \underline{\Pi}, \quad$ (c) $\frac{\partial E}{\partial t}=i \vec{k} \cdot \overrightarrow{\mathrm{Q}}$.

The tensors $\Pi_{i j}$ and $\sigma_{i j}$ are symmetric and have only six independent components as implied in (4. 2b). The variables $\sigma_{i j}$ and $q_{j}$ denote the viscous stress tensor and the thermal energy flux vector. The $\theta(\overrightarrow{\mathrm{k}})$, defined by (4.4), will be replaced later by

$$
T(\overrightarrow{\mathrm{k}}) \equiv \theta(\overrightarrow{\mathrm{k}}) / \rho_{0} C_{v}(k)
$$

whose average with respect to a perturbed distribution function yields the temperature in the conventional linearized hydrodynamic description of a fluid. ${ }^{16}$ Such an identification, however, is not needed for the present. The quantity $C_{v}(k)$ will be defined later (it will be identified as the specific heat at constant volume).

The average values of $\rho(\overrightarrow{\mathrm{k}}), E(\overrightarrow{\mathrm{k}})$, and $\Pi_{i j}(\overrightarrow{\mathrm{k}})$ are zero for $\vec{k} \neq 0$. When $\vec{k}=0$, we have

$$
\begin{aligned}
(1 / V)\langle\rho(0)\rangle & =\rho_{0}, \\
\frac{1}{V}\langle E(0)\rangle & =\frac{3}{2} \frac{\rho_{0}}{m \beta}+\frac{1}{2} \frac{\rho_{0}^{2}}{m^{2}} \int d^{3} R V(R) g(R), \\
\frac{1}{V}\left\langle\Pi_{i j}(0)\right\rangle & =\delta_{i j} P_{0} \\
& =\delta_{i j}\left(\frac{\rho_{0}}{m \beta}-\frac{\rho_{0}^{2}}{6 m^{2}} \int d^{3} R R \frac{d V(R)}{d R} g(R)\right) .
\end{aligned}
$$

shall from $\rho, E$, and $\Pi_{i j}$ whenever they are not zero, so that the state vector a will denote fluctuations.

Eight of the 14 components of the state vector a are even, and the remaining six are odd functions of particle velocities. Hence a can be decomposed into even and odd parts as

$$
\underline{\mathrm{a}}=\operatorname{col}\left[\underline{a}^{e}, 0\right]+\operatorname{col}\left[0, \underline{\mathrm{a}}^{0}\right] \text {. }
$$

Consequently, the static correlation matrix $\underline{\phi}=\left\langle\underline{\mathrm{a}}^{\dagger}\right\rangle$ splits into two disjoint submatrices as

$$
\underline{\phi}=\left[\begin{array}{cc}
\phi^{e} & 0 \\
0 & \underline{\phi}^{0}
\end{array}\right] \text {, }
$$

where

$$
\underline{\phi}^{e} \equiv\left\langle\underline{a}^{e} \underline{a}^{e \dagger}\right\rangle=\left[\begin{array}{ccc}
\left\langle\rho \rho^{*}\right\rangle & 0 & 0 \\
0 & \left\langle\theta \theta^{*}\right\rangle & 0 \\
0 & 0 & \left\langle\sigma \sigma^{\dagger}\right\rangle
\end{array}\right]
$$

and $\quad \underline{\phi}^{0} \equiv\left\langle\underline{a}^{0} \underline{a}^{0 \dagger}\right\rangle=\left[\begin{array}{cc}\left\langle\vec{J} \vec{J}^{\dagger}\right\rangle & 0 \\ 0 & \left\langle\vec{q} \vec{q}^{\dagger}\right\rangle\end{array}\right]$

The block diagonality of $\phi^{e}$ and $\phi^{0}$ is a consequence of the choice of the state variables as in (4.4),

(4.5), and (4.6), which imply the following orthogonality relations:

$$
\left\langle\rho \theta^{*}\right\rangle=\left\langle\sigma_{i j} \rho^{*}\right\rangle=\left\langle\sigma_{i j} \theta^{*}\right\rangle=\left\langle q_{i} J_{j}^{*}\right\rangle=0 .
$$

The static correlation functions appearing in Eqs. (4.13a) and (4.13b) will be discussed later.

The frequency matrix $(\underline{i \Omega})=\left\langle\underline{\dot{a}} \underline{a}^{\dagger}\right\rangle \cdot \phi^{-1}$ can be writ ten

$$
i \underline{\Omega}=\left[\begin{array}{cc}
0 & \left\langle\underline{\dot{a}}^{e} \underline{a}^{0 \dagger}\right\rangle \cdot \phi^{0^{-1}} \\
\left\langle\underline{\dot{a}}^{0} \underline{a}^{e \dagger}\right\rangle \cdot \underline{\phi}^{e-1}
\end{array}\right] .
$$

As a consequence of the conservation relations (4. 9), $f(0)=(1-P) \underline{\dot{a}}$ has only nine nonzero components:

$$
\underline{f}(0)=\operatorname{col}\left[0,0, f_{\mu}^{\sigma}, 0,0,0, f_{j}^{q}\right] .
$$

Therefore, the damping matrix $\varphi(t)$ is of the following form:

$$
\underline{\varphi}(t)=\left[\begin{array}{cccc}
0 & 0 & 0 & 0 \\
0 & \varphi^{\sigma \sigma}(t) & 0 & \varphi^{\sigma \alpha}(t) \\
0 & 0 & 0 & 0 \\
0 & \varphi^{\alpha \sigma}(t) & 0 & \varphi^{q \alpha}(t)
\end{array}\right],
$$

where $\varphi^{\sigma \sigma}$ and $\varphi^{q q}$ are $6 \times 6$ and $3 \times 3$ square matrices. The off-diagonal matrices $\varphi^{\alpha \sigma}(t)$ and $\varphi^{\sigma \alpha}$ are $3 \times 6$ and $6 \times 3$

Substituting $(4.15)$ and $(4.17)$ into the generalized Langevin equation discussed in Sec. II, we obtain the following set of equations:

$$
\begin{aligned}
& \dot{\rho}(t)=i \overrightarrow{\mathrm{k}} \cdot \overrightarrow{\mathrm{J}}(t) \text {, } \\
& \stackrel{\circ}{\vec{J}}(t)-\frac{\left\langle\dot{\vec{J}} \rho^{*}\right\rangle}{\left\langle\rho \rho^{*}\right\rangle} \rho(t)-\frac{\left\langle\dot{\vec{J}} \theta^{*}\right\rangle}{\left\langle\theta \theta^{*}\right\rangle} \theta(t)=i \overrightarrow{\mathbf{k}} \cdot \underline{\sigma}(t), \\
& \dot{\theta}(t)+\left\langle\theta \overrightarrow{\mathrm{J}}^{\dagger}\right\rangle \cdot\left\langle\overrightarrow{\mathrm{J}} \overrightarrow{\mathrm{J}}^{\dagger}\right\rangle^{-1} \cdot \overrightarrow{\mathrm{J}}(t)=i \overrightarrow{\mathrm{k}} \cdot \overrightarrow{\mathrm{q}}(t), \\
& \dot{\sigma}(t)-\left\langle\dot{\sigma} \overrightarrow{\mathrm{q}}^{\dagger}\right\rangle \cdot\left\langle\overrightarrow{\mathrm{q}} \overrightarrow{\mathrm{q}}^{\dagger}\right\rangle^{-1} \cdot \overrightarrow{\mathrm{q}}(t)+\int_{0}^{t} \underline{\varphi}^{\sigma \alpha}(t-u) \cdot \overrightarrow{\mathrm{q}}(u) d u \\
& -\left\langle\overrightarrow{\mathrm{Q}}^{\dagger}{ }^{\dagger}\right\rangle \cdot\left\langle\vec{J}^{\dagger}{ }^{\dagger}\right\rangle^{-1} \cdot \vec{J}(t)+\int_{0}^{t} \underline{\varphi}^{\sigma \sigma}(t-u) \cdot \underline{\sigma}(u) d u=\underline{f}^{\sigma}(t) \text {, } \\
& \dot{\overrightarrow{\mathrm{q}}}(t)-\frac{\left\langle\dot{\overrightarrow{\mathrm{q}}} \theta^{*}\right\rangle}{\left\langle\theta \theta^{*}\right\rangle} \theta(t)+\int_{0}^{t} \underline{\varphi}^{\alpha q}(t-u) \cdot \overrightarrow{\mathrm{q}}(u) d u \\
& -\left\langle\dot{\overrightarrow{\mathrm{q}}} \underline{\sigma}^{\dagger}\right\rangle \cdot\left\langle\underline{\sigma \sigma^{\dagger}}\right\rangle^{-1} \cdot \underline{\sigma}(t) \\
& +\int_{0}^{t} \underline{\varphi}^{q \sigma}(t-u) \cdot \underline{\sigma}(u) d u=\underline{f}^{q}(t) \text { 。 }
\end{aligned}
$$


Equations (4.18)-(4.22) describe the time evolution of the state variables $\rho, \theta, \sigma_{\mu}, J_{i}$, and $q_{i}$ exactly. We shall approximate them by neglecting the coupling between the viscous stress tensor and the energy flux vector and introducing the Markov assumption in (4.21) and (4.22). The first approximation is equivalent to setting $\left\langle\underline{\dot{\sigma}} \vec{q}^{\dagger}\right\rangle=0$ and $\phi^{\sigma q}$ $\equiv 0$ in $(4.21)$ and $(4.22)$, and the second to

$$
\begin{aligned}
& \int_{0}^{t} \underline{\varphi}^{\sigma \sigma}(t-u) \cdot \underline{\sigma}(u) d u \cong \underline{W}^{\sigma}(\overrightarrow{\mathrm{k}}) \cdot \underline{\sigma}(t), \\
& \int_{0}^{t} \underline{\varphi}^{q \alpha}(t-u) \cdot \overrightarrow{\mathrm{q}}(u) d u \tilde{=} \underline{W}^{\alpha}(\overrightarrow{\mathrm{k}}) \cdot \overrightarrow{\mathrm{q}}(t),
\end{aligned}
$$

$$
\text { where } \begin{aligned}
\underline{W}^{\sigma}(\overrightarrow{\mathrm{k}}) & \equiv \int_{0}^{\infty} d t \varphi^{\sigma \sigma}(t), \\
\underline{W}^{\alpha}(\overrightarrow{\mathrm{k}}) & =\int_{0}^{\infty} d t \varphi^{q \alpha}(t) .
\end{aligned}
$$

Furthermore, the following definitions and equalities will be introduced:

$$
\begin{aligned}
&\left\langle\dot{\vec{J}} \rho^{*}\right\rangle /\left\langle\rho \rho^{*}\right\rangle \equiv C_{L}^{2}(k) i \overrightarrow{\mathrm{k}} \\
&\left\langle\dot{\mathrm{J}} T^{*}\right\rangle /\left\langle T T^{*}\right\rangle \equiv C_{L}^{2}(k) \rho_{0} \beta_{L}(k) i \overrightarrow{\mathrm{k}}, \\
&(1 / V)\left\langle J_{i} J_{j}^{*}\right\rangle \equiv\left(\rho_{0} / \beta\right) \delta_{i j}, \\
& \frac{\left\langle\theta \theta^{*}\right\rangle}{\left\langle J_{1} J_{1}^{*}\right\rangle} \equiv \frac{\beta}{\rho_{0}}\left(\left\langle E E^{*}\right\rangle-\frac{\left|\left\langle E \rho^{*}\right\rangle\right|^{2}}{\left\langle\rho \rho^{*}\right\rangle}\right) \equiv T_{0} C_{v}(k) \\
&(\beta / V)\left\langle\dot{\sigma}_{i j} J_{m}^{*}\right\rangle \equiv i k_{n} \sum_{i j m n}(k), \\
&\left\langle\dot{\overrightarrow{\mathrm{q}}} \theta^{*}\right\rangle /\left\langle\theta \theta^{*}\right\rangle \equiv i \overrightarrow{\mathrm{k}} \cdot \underline{\Gamma}
\end{aligned}
$$

Then, we obtain the following approximate description:

$$
\begin{aligned}
& \dot{\rho}(t)=i k_{j} J_{j}(t), \\
& J_{j}(t)-C_{L}^{2}(k) i k_{j}\left[\rho(t)+\beta_{L}(k) \rho_{0} T(t)\right]=i k_{m} \delta_{j m}(t), \\
& \dot{\theta}(t)-\beta_{L}(k) C_{L}^{2}(k) T_{0} \dot{\rho}(t)=i k_{j} q_{j}(t), \\
& \dot{\sigma}_{\mu}(t)-\sum_{\mu \nu}(k) \epsilon_{\nu}(t)+W_{\mu \nu}^{\sigma}(k) \sigma_{\nu}(t)=f_{\mu}^{\sigma}(t), \\
& \dot{q}_{j}(t)-i k_{m} \Gamma_{j m} \theta(t)+W_{j m}^{q} q_{m}(t)=f_{j}^{q}(t),
\end{aligned}
$$

where $\epsilon_{m n}$ is the rate of strain tensor, i.e.,

$$
\epsilon_{m n}(k)=\left(i / 2 \rho_{0}\right)\left(k_{m} J_{n}+k_{n} J_{m}\right) \text { 。 }
$$

We shall discuss the physical implications of the various quantities appearing in this set.

The $C_{L}^{2}(k)$ introduced in (4.26a) can be defined also by

$$
\begin{aligned}
& C_{L}^{2}(k)=\left\langle\Pi_{33} \rho^{*}\right\rangle /\left\langle\rho \rho^{*}\right\rangle, \\
& C_{L}^{2}(k)=1 / \beta m S(k),
\end{aligned}
$$

where $S(k)$ is the structure factor defined by

$$
S(k)=1+\left(\rho_{0} / m\right) \int d^{3} R e^{i \overrightarrow{\mathrm{k}} \cdot \overrightarrow{\mathrm{R}}}[g(R)-1],
$$

$g(R)$ being the static pair correlation function. We will refer to $C_{L}(k)$ as the longitudinal isothermal speed of sound because one can also define a trans verse isothermal speed as

$$
\begin{aligned}
C_{T}^{2}(k) & \equiv\left\langle\Pi_{11} \rho^{*}\right\rangle /\left\langle\rho \rho^{*}\right\rangle=\left\langle\Pi_{22} \rho^{*}\right\rangle /\left\langle\rho \rho^{*}\right\rangle, \quad(4.30 \mathrm{a}) \\
C_{T}^{2}(k) & =\frac{1}{m \beta S(k)}\left(1-n \beta \int d^{3} R \frac{x^{2}-z^{2}}{R} \frac{d V}{d R} g(R) \frac{\sin \overrightarrow{\mathrm{k}} \cdot \overrightarrow{\mathbf{R}}}{\overrightarrow{\mathrm{k}} \cdot \overrightarrow{\mathrm{R}}}\right. \\
& \left.-\frac{n^{2} \beta}{2} \int d^{3} R \frac{x^{2}-z^{2}}{R} \frac{d V}{d R} g(\overrightarrow{\mathrm{R}}, \overrightarrow{\mathrm{k}}) \frac{1-e^{-i \overrightarrow{\mathrm{k}} \cdot \overrightarrow{\mathrm{R}}}}{i \overrightarrow{\mathrm{k}} \cdot \overrightarrow{\mathrm{R}}}\right),
\end{aligned}
$$

where $g(\vec{R}, \vec{k})$ is the Fourier transform of the threeparticle distribution function $g(\vec{R}, \vec{S})$ with respect to $\overrightarrow{\mathrm{S}}$, i. e.,

$$
\begin{gathered}
n^{3} g(\overrightarrow{\mathrm{R}}, \overrightarrow{\mathrm{k}}) \equiv \frac{1}{V}\left\langle\sum_{\alpha, \beta, \gamma} \delta\left(\overrightarrow{\mathrm{R}}-\overrightarrow{\mathrm{x}}^{\alpha \beta}\right) e^{\left.i \overrightarrow{\mathrm{R}} \cdot \overrightarrow{\mathrm{x}}^{\alpha \beta}\right\rangle}\right. \\
(\beta, \gamma \neq \alpha ; \beta \neq \gamma) .
\end{gathered}
$$

In the isotropic limit where $k a \ll 1, a$ being the mean linear force range, $\overrightarrow{\mathrm{k}} \cdot \overrightarrow{\mathrm{R}} \ll 1$ and $C_{L}^{2}=C_{T}^{2}$. In this limit, the distinction between the longitudinal and the transverse speeds becomes unnecessary. The conventional isothermal speed of sound $C_{0}(k)$ is defined by ${ }^{16,21}$

$$
C_{0}^{2}(k)=\left.\frac{\partial P}{\partial \rho}\right|_{T}=\frac{1}{3} \frac{\left\langle\left(\operatorname{Tr} \Pi_{i j}\right) \rho^{*}\right\rangle}{\left\langle\rho \rho^{*}\right\rangle},
$$

where $P$ is the thermodynamic part pressure ${ }^{16,21}$ $p=\frac{1}{3} \operatorname{Tr} \Pi_{i j}$ (the nonthermodynamic part, by definition, has no projection on $\rho$ and $T$ ). Thus, the longitudinal and transverse isothermal speeds are related to $C_{0}(k)$ by

$$
C_{0}^{2}(k)=\frac{1}{3}\left[C_{L}^{2}(k)+2 C_{T}^{2}(k)\right] .
$$

In the isotropic limit, we have $C_{0}(k)=C_{L}(k)$. We may note that only the longitudinal isothermal speed is completely determined by the structure function $S(k)$ when $k$ is not small.

The quantity $\beta_{L}(k)$ in $(4.26 \mathrm{~b})$ can be defined equivalently by

$$
\beta_{L}(k) \equiv\left[1 / \rho_{0} C_{L}^{2}(k)\right]\left\langle\Pi_{33} T^{*}\right\rangle /\left\langle T T^{*}\right\rangle
$$

(use $i k\left\langle\Pi_{33} T^{*}\right\rangle=\left\langle\dot{J}_{3} T^{*}\right\rangle$ ). We refer to $\beta_{L}(k)$ as the longitudinal thermal-expansion coefficient for the following reasons. Similar to (4.30a) we can also define a transverse thermal-expansion coefficient as

$$
\beta_{T}(k) \equiv\left[1 / \rho_{0} C_{T}^{2}(k)\right]\left\langle\Pi_{11} T^{*}\right\rangle /\left\langle T T^{*}\right\rangle .
$$

(Note that $\left\langle\Pi_{11} T^{*}\right\rangle=\left\langle\Pi_{22} T^{*}\right\rangle$.) The conventional definition of the thermal-expansion coefficient $\beta_{0}$ as a derivative of the thermodynamic part of pres$\operatorname{sure}^{16,21}$ is 


$$
\begin{aligned}
\beta_{0}(k) & \equiv \frac{1}{\beta_{0} C_{0}^{2}(k)} \frac{1}{3} \frac{\left\langle\left(\operatorname{Tr} \Pi_{i j}\right) T^{*}\right\rangle}{\left\langle T T^{*}\right\rangle} \\
& \left.\equiv \frac{1}{\rho_{0} C_{0}^{2}(k)} \frac{\partial P}{\partial T}\right|_{\rho} .
\end{aligned}
$$

Then, $\beta_{0}(k)$ is related to $\beta_{L}(k)$ and $\beta_{T}(k)$ by

$$
\beta_{0}(k)=\frac{\beta_{L}(k) C_{L}^{2}(k)+2 \beta_{T}(k) C_{T}^{2}(k)}{C_{L}^{2}(k)+2 C_{T}^{2}(k)} .
$$

In the isotropic limit defined above $\beta_{0}(k)=\beta_{L}(k)$ $=\beta_{T}(k)$. Since they reduce to the derivative of the pressure with respect to temperature as in (4.33) in the limit of $k \rightarrow 0, \beta_{L}(k)$ and $\beta_{T}(k)$ are identified as thermal-expansion coefficients.

The definition of $C_{v}(k)$ in $(4.26 \mathrm{~d})$ is identical to that by Schofield. ${ }^{21}$ It is identified as the $k$-dependent specific heat at constant volume. Its expression in terms of the multiparticle distribution functions is given in the cited reference [Eq. (50) of Ref. 21].

The symmetric tensor $\sum_{u v}$ introduced in $(4.26 \mathrm{e})$ can be defined alternatively by

$$
\begin{aligned}
& \Sigma_{u v}=(\beta / V)\left\langle\sigma_{\mu} \sigma_{\nu}^{*}\right\rangle, \\
& \Sigma_{\mu \nu}=C_{\mu \nu}-\frac{\beta}{V}\left(\frac{\left\langle\Pi_{\mu}, o^{*}\right\rangle\left\langle\rho \Pi_{\nu}^{*}\right\rangle}{\left\langle\rho \rho^{*}\right\rangle}\right. \\
&\left.+\frac{\left\langle\Pi_{\mu} T^{*}\right\rangle\left\langle T \Pi_{\nu}^{*}\right\rangle}{\left\langle T T^{*}\right\rangle}\right),
\end{aligned}
$$

where $C_{\mu \nu}$ is the elastic-moduli tensor defined by

$$
C_{\mu \nu} \equiv(\beta / V)\left\langle\Pi_{\mu} \Pi_{\nu}^{*}\right\rangle \text {. }
$$

The elements $C_{44}, C_{31}$, and $C_{33}$ have been computed by Schofield ${ }^{21}$ as functions of $k$ in terms of the twoparticle distribution function. One can show that $C_{\mu \nu}(k)$ has the following form:

$$
\underline{C}(k)=\left[\begin{array}{cccccc}
C_{11} & C_{12} & C_{13} & & & \\
C_{12} & C_{11} & C_{13} & & 0 & \\
C_{13} & C_{13} & C_{33} & & & \\
& & & C_{44} & & 0 \\
& 0 & & & C_{44} & \\
& & & 0 & & C_{66}
\end{array}\right],
$$

with the following relation:

$$
C_{11}(k)-C_{12}(k)=2 C_{66}(k) \text {. }
$$

This relation which can be verified directly is a consequence of the isotropy in a plane perpendicular to $\overrightarrow{\mathrm{k}}$. In the limit of $k \rightarrow 0$, one finds in addition to $(4.36 \mathrm{~b})$

$$
C_{11}=C_{33}, \quad C_{13}=C_{12}, \quad C_{33}-C_{13}=2 C_{44} .
$$

Hence, $\underline{C}(k)$ has in general only five distinct nonzero elements, i. e., $C_{11}, C_{12}, C_{13}, C_{33}$, and $C_{44}$. In the isotropic limit there are only two: $C_{13}$ and $C_{44}$.

Verifying

$$
\begin{aligned}
& \left\langle\Pi_{i j} \rho^{*}\right\rangle=\delta_{i j}\left\langle\Pi_{i i} \rho^{*}\right\rangle, \\
& \left\langle\Pi_{i j} T^{*}\right\rangle=\delta_{i j}\left\langle\Pi_{i i} T^{*}\right\rangle,
\end{aligned}
$$

and using (4.36a) in (4.34b) we obtain the form of $\Sigma_{\mu \nu}$ as

$$
\underline{\Sigma}=\left[\begin{array}{cccccc}
\Sigma_{11} & \Sigma_{12} & \Sigma_{13} & & & \\
\Sigma_{12} & \Sigma_{11} & \Sigma_{13} & & 0 & \\
\Sigma_{13} & \Sigma_{13} & \Sigma_{33} & & & \\
& & & \Sigma_{44} & & 0 \\
& 0 & & & \Sigma_{44} & \\
& & & 0 & & \Sigma_{66}
\end{array}\right] \text {, }
$$

where $\Sigma_{11}=C_{11}-\rho_{0}\left(C_{T}^{4} / C_{L}^{2}\right) \gamma_{T}$,

$$
\Sigma_{12}=C_{12}-\rho_{0}\left(C_{T}^{4} / C_{L}^{2}\right) \gamma_{T},
$$

$\Sigma_{33}=C_{33}-\rho_{0} C_{L}^{2} \gamma_{L}$,

$$
\Sigma_{13}=C_{13}-\rho_{0} C_{T}^{2} \gamma_{T L} \quad \text {. }
$$

In (4.39), we have introduced

$$
\begin{aligned}
\gamma_{T}(k) & \equiv 1+\left[T_{0} C_{L}^{2}(k) \beta_{T}^{2}(k) / C_{V}(k)\right], \\
\gamma_{L}(k) & \equiv 1+\left[T_{0} C_{L}^{2}(k) \beta_{L}^{2}(k) / C_{V}(k)\right], \\
\gamma_{T L}(k) & \equiv 1+\left[T_{0} C_{L}^{2}(k) \beta_{L}(k) \beta_{T}(k) / C_{V}(k)\right] .
\end{aligned}
$$

In the isotropic limit, $\gamma_{T}(k), \gamma_{L}(k)$, and $\gamma_{T L}(k)$ become equal to

$$
\gamma=1+\left(T_{0} C_{0}^{2} \beta_{0}^{2} / C_{V}\right),
$$

which is the conventional ratio of the specific heats. It is interesting to note that $\Sigma_{11}(k)-\Sigma_{12}(k)=2 \Sigma_{66}(k)$ holds also for $\Sigma_{\mu \nu}$ for all values of $k$. In the isotropic limit we obtain

$$
\Sigma_{12}=\Sigma_{13}, \quad \Sigma_{11}=\Sigma_{33}, \quad \Sigma_{33}-\Sigma_{13}=2 \Sigma_{44} .
$$

The matrix $\Gamma(k)$ introduced in (4.26f) has the following alternative definition:

$$
\begin{aligned}
\Gamma_{i j}(k) & \equiv\left\langle q_{i} q_{j}^{*}\right\rangle /\left\langle\theta \theta^{*}\right\rangle \\
& =\left\langle\theta \theta^{*}\right\rangle^{-1}\left[\left\langle Q_{i} Q_{j}^{*}\right\rangle-\left(\beta / \rho_{0} V\right)\left|\left\langle Q_{i} J_{j}^{*}\right\rangle\right|^{2}\right] .
\end{aligned}
$$

It can be shown that $\left\langle q_{i} q_{j}^{*}\right\rangle$ is diagonal with the elements $\left\langle q_{1} q_{1}^{*}\right\rangle=\left\langle q_{2} q_{2}^{*}\right\rangle$ and $\left\langle q_{3} q_{3}^{*}\right\rangle$. Hence

$$
\underline{\Gamma}=\operatorname{diag}\left[\Gamma_{T}, \Gamma_{T}, \Gamma_{L}\right] \text {, }
$$

where

$$
\begin{aligned}
& \Gamma_{T}(k) \equiv\left\langle\theta \theta^{*}\right\rangle^{-1}\left[\left\langle Q_{1} Q_{1}^{*}\right\rangle-\left(\beta / \rho_{0} V\right)\left|\left\langle Q_{1} J_{1}^{*}\right\rangle\right|^{2}\right], \\
& \Gamma_{L}(k) \equiv\left\langle\theta \theta^{*}\right\rangle^{-1}\left[\left\langle Q_{3} Q_{3}^{*}\right\rangle-\left(\beta / \rho_{0} V\right)\left|\left\langle E \Pi_{33}^{*}\right\rangle\right|^{2}\right],
\end{aligned}
$$

where $\left\langle Q_{3} J_{3}^{*}\right\rangle=\left\langle E \Pi_{33}^{*}\right\rangle$ from stationarity. We shall relate $\underline{\Gamma}$ later to the thermal diffusivity tensor.

Relaxation Frequencies

We now focus our attention upon (4.27d) and 
(4. 27e) which are generalizations of Maxwell's model for the relaxation of the viscous stress tensor, and Fourier's law. They both contain the $k$ dependent relaxation frequency matrices $W^{\sigma}$ and $W^{q}$ which are defined by $(4.24)$ and $(4.25)$, respectively. Although the elements of these matrices can be obtained in principle from their definitions [cf. (4.25)], we are forced in applications to model them as explained below. [A direct evaluation of $W^{\sigma}$ and $W^{q}$ using (4.24) and (4.25) is equivalent to solving Liouville's equation with the reduced Liouville operator $(1-P) L$, and thus not yet possible.]

We first consider $W^{\sigma}$ appearing in (4.27d). The mean of this equation reduces in steady state to

$$
W_{\mu \nu}^{\sigma}\left\langle\sigma_{\nu}\right\rangle=\Sigma_{\mu \nu}\left\langle\epsilon_{\nu}\right\rangle \text {, }
$$

where $\left\langle\sigma_{\nu}\right\rangle$ and $\left\langle\epsilon_{\nu}\right\rangle$ are the expected values of $\sigma_{\mu}$ and $\epsilon_{\nu}$ (recall that $\left\langle f_{\mu}^{\sigma}\right\rangle=0$ in the linear approxima$\left.\operatorname{tion}^{16}\right)$. Since the linear relation between the viscous stress tensor and the rate of the strain tensor defines the viscosity tensor $\eta_{i j m n}$ according to

$$
\left\langle\sigma_{i j}\right\rangle=\eta_{i j m n}\left\langle\epsilon_{m n}\right\rangle \quad(m, n=1,2,3),
$$

we have

$$
\Sigma_{\mu \nu}=W_{\mu \lambda}^{\sigma} \eta_{\lambda \nu}
$$

We shall use $(4.44 \mathrm{c})$ as a guide in modelling the components of $W_{\mu \nu}^{\sigma}$. The form of $\eta_{i j m n}$ consistent with the cylindrical symmetry about $\vec{k}$ is

$$
\underline{\eta}=\left[\begin{array}{cccccc}
\eta_{11} & \eta_{12} & \eta_{13} & & & \\
\eta_{12} & \eta_{11} & \eta_{13} & & 0 & \\
\eta_{13} & \eta_{13} & \eta_{33} & & & \\
& & & \eta_{44} & & 0 \\
& 0 & & & \eta_{44} & \\
& & & 0 & & \eta_{66}
\end{array}\right]
$$

with the condition

$$
\eta_{11}(k)-\eta_{12}(k)=2 \eta_{66}(k),
$$

which implies isotropy in the plane perpendicular to $\vec{k}$. In order to ensure isotropy in all directions in the limit of $k \rightarrow 0$ we must also require

$$
\begin{aligned}
\eta_{33}(k)- & \eta_{13}(k) \\
\eta_{13}(k) & \rightarrow \eta_{12}(k), \\
\eta_{33}(k) & \rightarrow \eta_{11}(k), \text { as } k \rightarrow 0 .
\end{aligned}
$$

Thus, the number of independent components of $\eta_{\mu \nu}$ is five (i.e., $\eta_{11}, \eta_{12}, \eta_{13}, \eta_{33}$, and $\eta_{44}$ ) for arbitrary values of $k$, and only two (i.e., $\eta_{13}$ and $\left.\eta_{44}\right)$ in the limit of $k \rightarrow 0$. In this limit, $(4.44 \mathrm{~b}) \mathrm{re}-$ duces to

$$
\left\langle\sigma_{i j}\right\rangle=\left(\eta_{13}+\frac{2}{3} \eta_{44}\right) \frac{\partial\left\langle v_{m}\right\rangle}{\partial x_{m}} \delta_{i j}
$$

$$
+\eta_{44}\left(\frac{\partial\left\langle v_{i}\right\rangle}{\partial x_{j}}+\frac{\partial\left\langle v_{j}\right\rangle}{\partial x_{i}}-\frac{2}{3} \delta_{i j} \frac{\partial\left\langle v_{m}\right\rangle}{\partial x_{m}}\right),
$$

which is the conventional form, $\eta_{44}$ and $\left(\eta_{13}+\frac{2}{3} \eta_{44}\right)$ being the shear and bulk viscosity coefficients.

Using the form of $\Sigma_{\mu \nu}$ in (4.38a) and (4.44c) we can obtain a form for the relaxation frequency matrix:

$$
\underline{W}^{\sigma}=\left[\begin{array}{llllll}
\omega_{11} & \omega_{12} & \omega_{13} & & & \\
\omega_{12} & \omega_{11} & \omega_{13} & & 0 & \\
\omega_{13} & \omega_{13} & \omega_{33} & & & \\
& & & \omega_{44} & & 0 \\
& 0 & & & \omega_{44} & \\
& & & 0 & & \omega_{66}
\end{array}\right] \text {, }
$$

with the following restrictions:

$$
\begin{aligned}
& \omega_{13}\left(\Sigma_{33}-\Sigma_{12}-\Sigma_{11}\right)=\Sigma_{13}\left(\omega_{33}-\omega_{12}-\omega_{11}\right), \\
& \omega_{11}-\omega_{12}=\omega_{66}, \\
& \omega_{13}-\omega_{12}, \omega_{44}-\omega_{11}-\omega_{12}, \quad \text { as } k \rightarrow 0 .
\end{aligned}
$$

There are four independent relaxation frequencies [i. e., $\omega_{11}(k), \omega_{12}(k), \omega_{13}(k)$, and $\omega_{44}(k)$ ] for arbitrary values of $k$, and two (i. e., $\omega_{33}$ and $\omega_{13}$ ) for $k=0$. Although it is possible to obtain the time correlation functions in terms of these relaxation frequencies we prefer working with a simpler model in which $\omega_{12}=\omega_{13}=0$ for all $k$. It will be seen later that this choice of relaxation frequencies eliminates the coupling between the components of the stress tensor during their relaxation. Setting $\omega_{12} \equiv \omega_{13} \equiv 0$ and denoting $\omega_{11}$ and $\omega_{44}$ by $\omega_{L}$ and $\omega_{S}$, respectively, in (4.47) and (4.48), we find

$$
\underline{W}^{\sigma}=\operatorname{diag}\left(\omega_{L}, \omega_{L}, \omega_{L}, \omega_{S}, \omega_{S}, \omega_{L}\right) .
$$

Thus, we have to model only two frequencies, $\omega_{L}(k)$ and $\omega_{S}(k)$, which satisfy $\omega_{L}(0)=\omega_{S}(0)$.

We next consider $W^{q}$ in (4.27e) whose mean reduces in steady state to

$$
W_{n j}^{q}\left\langle q_{j}\right\rangle=\Gamma_{n j} i k_{j}\langle\theta\rangle \text {. }
$$

Comparing this equation to

$$
\left\langle q_{j}\right\rangle=D_{j m} i k_{m}\langle\theta\rangle,
$$

where $D_{i j}$ is the thermal diffusivity tensor, we obtain

$$
\underline{W}^{q} \cdot \underline{D}=\underline{\Gamma} .
$$

Since $\underline{\Gamma}$ is diagonal (cf. 4.43a), we choose

$$
\underline{W}^{q}=\operatorname{diag}\left(\omega_{1}, \omega_{1}, \omega_{3}\right) \text {, }
$$

which implies a diagonal thermal diffusivity tensor. Denoting the elements of $\underline{D}$ by $\left(D_{T}, D_{T}, D_{L}\right)$, we find

$$
\begin{aligned}
& D_{T}(k)=\Gamma_{T}(k) / \omega_{1}(k), \\
& D_{L}(k)=\Gamma_{L}(k) / \omega_{3}(k) .
\end{aligned}
$$


In the limit of $k \rightarrow 0, \Gamma_{L}(k) \rightarrow \Gamma_{T}(k)$. Hence we must require also $\omega_{1}(0)=\omega_{3}(0)$. In the isotropic limit $(4.50 \mathrm{~b})$ reduces to the conventional Fourier's law

$$
\langle\vec{q}\rangle=-D \nabla\langle\theta\rangle,
$$

where $D$ is the thermal diffusivity coefficient given by

$$
D=D_{L}(0)=D_{T}(0) .
$$

\section{Transverse Current Correlations}

The projection of $\vec{J}(\vec{k})$ on a fixed axis perpendicular to $\overrightarrow{\mathrm{k}}$ satisfies the following equations obtained from (4.27b) and (4.27d):

$$
\begin{aligned}
& \dot{J}_{1}(t)=i k \sigma_{4}(t), \\
& \dot{\sigma}_{4}(t)-\left(i k / \rho_{0}\right) C_{44}(k) J_{1}(t)+\omega_{S}(k) \sigma_{4}(t)=f_{4}^{\sigma}(t) .
\end{aligned}
$$

Multiplying these equations by $J_{1}^{*}$, taking the thermal averages of the resulting equations, and Laplace-transforming, we obtain the transverse current spectral density as

$$
R_{T}(k, \omega) \cong \operatorname{Re}\left[i \omega+\left(k^{2} / \rho_{0}\right) \eta_{S}(k, i \omega)\right]^{-1},
$$

where $\eta_{S}(k, i \omega) \equiv C_{44}(k) /\left[i \omega+\omega_{S}(k)\right]$.

Using (4.44c) we find that

$$
\eta_{S}(k, 0)=\eta_{44}(k) \text {, }
$$

which, according to $(4.46 \mathrm{c})$, indicates that $\eta_{S}(0,0)$ is the conventional shear viscosity. Thus, $\eta_{s}(k, i \omega)$ can be identified as the $k$ - and $\omega$-dependent shear viscosity.

A comparison of (4.53) with (3.10) and (3.11) indicates that

$$
\eta_{T}(k, i \omega) \approx \eta_{S}(k, i \omega),
$$

i. e., the transverse viscosity defined by (3.11) can be interpreted approximately as the $k$ - and $\omega-$ dependent shear viscosity. Equation (4.54a) further implies that

$$
\omega_{T}(k) \cong \omega_{S}(k) \text {. }
$$

[Here, we should remember that the projection operators involved in the definitions of $\omega_{T}(k)$ (i.e., in the two-component description) and $\omega_{S}(k)$ (i. e., in the hydrodynamic description) are different. ] Thus, we can use the same expression for the $k$ dependence of $\omega_{S}(k)$ as that of $\omega_{T}(k)$ in (3.20).

\section{Longitudinal Current Correlation}

The component of $\vec{J}(\vec{k})$ parallel to $\underline{k}$ satisfies the following set of equations obtained from (4.27):

$$
\begin{aligned}
\stackrel{\circ}{\rho}(t) & =i k J_{3}(t), \\
\dot{J}_{3}(t)-i k C_{L}^{2}\left[\rho(t)+\beta_{L} \rho_{0} T(t)\right] & =i k \sigma_{3}(t),
\end{aligned}
$$

$$
\begin{aligned}
\dot{\theta}(t)-\beta_{L} C_{L}^{2} T_{0} \dot{\rho}(t) & =i k q_{3}, \\
\dot{\sigma}_{3}(t)-\left(i k / \rho_{0}\right) \Sigma_{33} J_{3}(t)+\omega_{L} \sigma_{3}(t) & =f_{3}^{\sigma}(t), \\
\dot{q}_{3}(t)+\omega_{3}\left[q_{3}(t)-i k D_{L} \theta(t)\right] & =f_{3}^{a}(t) .
\end{aligned}
$$

In (4.56) we have made use of the modeled form of the relaxation frequency matrices $W^{\sigma}$ and $W^{q}$.

Equations (4.55) are exact, and equivalent to the conservation laws (4.9). Multiplying these equations by $J_{3}^{*}(0)$, taking thermal averages, and Laplace-transforming the resulting equations with respect to time, we obtain for the longitudinal current spectrum, i.e.,

$$
R_{L}(k, \omega) \equiv \int_{0}^{\infty} d t \frac{\left\langle J_{3}(t) J_{3}^{*}(0)\right\rangle}{\left\langle J_{3} J_{3}^{*}\right\rangle} \cos \omega t,
$$

the following expression:

$$
\begin{aligned}
R_{L}(k, \omega)= & \operatorname{Re}\left(i\left[\omega-k^{2} C_{L}^{2}(k) / \omega\right]\right. \\
& \left.+\frac{k^{2}}{\rho_{0}} \eta_{L}(k, i \omega)+\frac{k^{2} C_{L}^{2}(k)\left[\gamma_{L}(k)-1\right]}{i \omega+k^{2} \delta(k, i \omega)}\right)^{-1},
\end{aligned}
$$

where we have defined

$$
\begin{aligned}
\eta_{L}(k, i \omega) & \equiv \frac{\rho_{0}}{i k} \frac{\left\langle\sigma_{3}(i \omega) J_{3}^{*}\right\rangle}{\left\langle J_{3}(i \omega) J_{3}^{*}\right\rangle}, \\
\delta(k, i \omega) & \equiv \frac{1}{i k} \frac{\left\langle q_{3}(i \omega) J_{3}^{*}\right\rangle}{\left\langle\theta(i \omega) J_{3}^{*}\right\rangle} .
\end{aligned}
$$

It is clear that $(4.58)$ is exact provided $\eta_{L}$ and $\delta$ are determined from (4.59) and (4.60) exactly. We shall use (4.56) to compute $\eta_{L}$ and $\delta$, and thereby obtain an approximate expression for $R_{L}(k, \omega)$ :

$$
\begin{aligned}
\eta_{L}(k, i \omega) & =\Sigma_{33}(k) /\left[i \omega+\omega_{L}(k)\right], \\
\eta_{L}(k, i \omega) & =\frac{C_{33}(k)-\rho_{0} \gamma_{L}(k) C_{L}^{2}(k)}{i \omega+\omega_{L}(k)}, \\
\delta(k, i \omega) & =\left\{D_{L}(k) /\left[i \omega+\omega_{3}(k)\right]\right\} \omega_{3}(k),
\end{aligned}
$$

where we have used $(4.39 \mathrm{c})$ to replace $\Sigma_{33}(k)$.

It is interesting to see the connection between $\eta_{L}(k, i \omega)$ and the $k$ - and $\omega$-dependent bulk viscosity $\eta_{B}(k, i \omega)$. The latter is defined by

$$
\begin{aligned}
\eta_{B}(k, i \omega) & \equiv \frac{\rho_{0}}{i k} \frac{1}{3} \frac{\left\langle\operatorname{Tr} \sigma_{i j}(i \omega) J_{3}^{*}\right\rangle}{\left\langle J_{3}(i \omega) J_{3}^{*}\right\rangle} \\
& =\frac{1}{3}\left[\Sigma_{33}(k)+2 \Sigma_{13}(k)\right] /\left[i \omega+\omega_{L}(k)\right] .
\end{aligned}
$$

The expression in (4.63) is obtained by summing (4. 27d) for $\mu=1,2$, and 3 . When $\omega=0$ and $k=0$, (4.63) reduces to

$$
\eta_{B}(0,0)=\eta_{13}(0)+\frac{2}{3} \eta_{44}(0),
$$

where we have used $\eta_{33}=\Sigma_{33} / \omega_{L}$ and $\eta_{13}=\Sigma_{13} / \omega_{L}$ [cf. $(4.44 \mathrm{c})]$, and $\eta_{33}(0)=\eta_{13}(0)+2 \eta_{44}(0)$. We find from $(4.46 \mathrm{c})$ that $\eta_{B}(0,0)$ is indeed conventional 
bulk viscosity.

We can express $\eta_{L}(k, i \omega)$ in $(4.61 \mathrm{~b})$ in terms of $\eta_{B}(k, i \omega)$ as

$$
\eta_{L}(k, i \omega)=\eta_{B}(k, i \omega)+\frac{2}{3} \frac{\Sigma_{33}(k)-\Sigma_{13}(k)}{i \omega+\omega_{L}(k)} .
$$

In the limit of $k \rightarrow 0$, we have $\Sigma_{33}(k)-\Sigma_{13}(k) \rightarrow 2 \Sigma_{44}(k)$ and $\omega_{L}(k) \rightarrow \omega_{44}(k) \approx \omega_{S}(k)$. Hence, $(4.65)$ reduces to the conventional form

$$
\eta_{L}(0, i \omega)=\eta_{B}(0, i \omega)+\frac{4}{3} \eta_{S}(0, i \omega)
$$

\section{ANALYSIS OF RAHMAN'S DATA}

We have analyzed the computer data of Rahman ${ }^{2}$. using

$$
R_{L}(k, \omega)=\operatorname{Re}\left[i\left(\omega-\frac{k^{2} C_{L}^{2}(k)}{\omega}\right)+\frac{k^{2}}{\rho_{0}} \eta_{L}(k, i \omega)\right]^{-1},
$$

which is obtained from (4.58) by ignoring the last term in the curly bracket. We have found that this term, which represents the thermal effects, becomes insignificant for large values of $k$ (e.g. , $k>1 \AA^{-1}$ for argon) as compared to $\left(k^{2} / \rho_{0}\right) \eta_{L}$ in (4.58). Substituting $\eta_{L}(k, i \omega)$ from $(4.61 \mathrm{~b})$ into (5. 1) we find

$$
R_{L}(k, \omega)=\frac{\omega^{2} k^{2} \omega_{L}(k)\left[C_{33}(k)-\rho_{0} \gamma_{L}(k) C_{L}^{2}(k)\right] / \rho_{0}}{\omega^{2}\left\{\omega^{2}-\left(k^{2} / \rho_{0}\right)\left[C_{33}(k)-\left(\gamma_{L}(k)-1\right) \rho_{0} C_{L}^{2}(k)\right]\right\}^{2}+\omega_{L}^{2}(k)\left[\omega^{2}-k^{2} C_{L}^{2}(k)\right]^{2}}
$$

where $C_{33}(k)$ can be calculated from $(4.35)$ as

$C_{33}(k)=n\left(\frac{3}{\beta}+n \int d^{3} R \frac{d^{2} V}{d z^{2}} g(R) \frac{1-\cos k z}{k^{2}}\right)$

[see Fig. 1 for the variation of $C_{33}(k)$ with $k$ ]. The $k$ dependence of $\gamma_{L}(k)$ defined in $(4.40 \mathrm{~b})$ is difficult to determine because its calculation involves multiparticle distribution function. In the analysis of Rahman's data we set $\gamma_{L}(k) \cong 1$ for the sake of simplicity. Then, the only unknown function in (5.2) was $\omega_{L}(k)$. Its value at $k=0$ was estimated using

$$
\omega_{L}(0)=\left[C_{33}(0)-\rho_{0} C_{L}^{2}(0) \gamma\right] /\left(\eta_{B}+\frac{4}{3} \eta_{S}\right),
$$

where $\eta_{B}$ and $\eta_{S}$ are the conventional bulk and shear viscosities, respectively. We note that

$$
\lim _{k \rightarrow 0} C_{33}(k)=K_{\infty}+\frac{4}{3} G_{\infty},
$$

where $G_{\infty}$ was defined in Sec. III and $K_{\infty}$ is the bulk modulus in Zwanzig's notation. ${ }^{23}$ The value of $C_{33}(0)$ was read from Fig. 1 , and that of $C_{L}^{2}(0)$ (the ordinary isothermal speed of sound) was calculated from (4.29b). [Rahman has also calculated $S(k)$.]

To model the large- $k$ behavior of $\omega_{L}(k)$ we consider the ideal gas,

$$
R_{L}^{I G}(k, \omega)=\beta m \frac{\omega^{2}}{k^{2}}\left(\frac{\pi \beta m}{2 k^{2}}\right)^{1 / 2} e^{-\beta m \omega^{2} / 2 k 2},
$$

which has a maximum at $\omega_{m}^{2}(k)=2 k^{2} / \beta m$. Requiring that the frequency at which (5.2) attains its maximum for a fixed $k$ approach $\left(2 k^{2} / \beta m\right)$ for large $k$ and using the same interpolation formula as in (3. 20) we obtain the following expression for $\omega_{L}(k)$ :

$$
\begin{aligned}
\omega_{L}^{2}(k)= & \frac{8}{3 \rho_{0}} k^{2}\left(C_{33}(k)-\rho_{0} C_{L}^{2}(k)-\frac{\rho_{0}}{\beta m}\right) \\
& +\left[\omega_{L}^{2}(0)-\frac{8 k^{2}}{3 \rho_{0}}\left(C_{33}(k)-\rho_{0} C_{L}^{2}(k)-\frac{2 \rho_{0}}{\beta m}\right)\right. \\
& \left.\times\left(1+k^{2} / k_{0}^{2}\right)^{-1}\right],
\end{aligned}
$$

and where $\omega_{L}(0)$ is given by $(5.4)$. The variation of $\omega_{L}(k)$ is shown in Fig. $2\left(k_{0}=1.5 \AA^{-1}\right.$ as in the case of transverse correlations).

It is interesting to compare again $R_{L}(k, \omega) / \omega^{2}$ from (5.2) and $R_{L}^{I G}(k, \omega) / \omega^{2}$ from (5.6) for $k \rightarrow \infty$ and $\omega \rightarrow 0$, as in the case of transverse current power spectral density. Using $C_{L}^{2}(k) \rightarrow(1 / \beta m)$ and $C_{33}(k) \rightarrow\left(3 \rho_{0} / \beta m\right)$ as $k \rightarrow \infty$, we find $R_{L}(k, 0) / \omega^{2}$ $\rightarrow(\beta m)^{3 / 2} \sqrt{ }\left(\frac{3}{2}\right) / k^{3}$ and $R_{L}^{I G}(k, 0) / \omega^{2} \rightarrow(\beta m)^{3 / 2}$ $\times \sqrt{ }\left(\frac{1}{2} \pi\right) / k^{3}$. Thus, the approximate formula for $R_{L}(k, \omega)$ yields the ideal gas limit for small $\omega$ and large $k$ within a factor $\sqrt{ }(3 / \pi) \approx 0.98$.

The curves in Figs. 6 and 7 are calculated using (5.2) and (5.7). The agreement between Rahman's computer results and the theoretical curves are remarkably good.

The thermal effects dominate for small values of $k$ such as those involved in light scattering. ${ }^{12}$ We then approximate $(4.58)$ as

$$
\begin{aligned}
R_{L}(k, \omega)= & \operatorname{Re}\left\{i\left[\omega-k^{2} C_{L}^{2}(0) / \omega\right]+\left(k^{2} / \rho_{0}\right) \eta_{L}(0,0)\right. \\
& \left.+C_{L}^{2}(0) k^{2}(\gamma-1) /\left(i \omega+k^{2} D\right)\right\}^{-1},
\end{aligned}
$$

which is the result corresponding to the conventional hydrodynamic description with constant transport parameters. It appears that one has to include both the thermal effects and the dependence of $\eta_{L}$ on $k$ and $\omega$ for the intermediate values of $k$. Since there are no computer results for such val- 

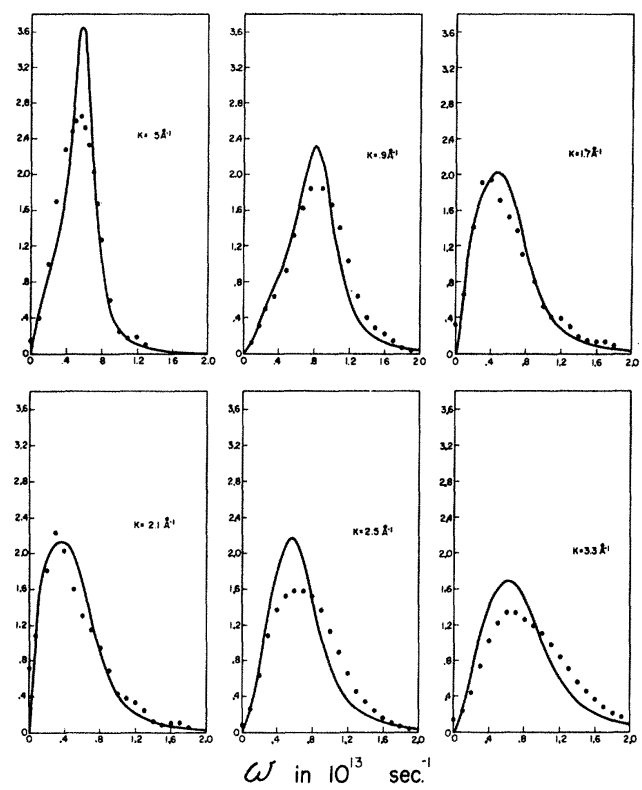

FIG. 6. The longitudinal current-current correlation function versus $\omega$ for various $k$. The points are from Rahman.

ues of $k$ we have not attempted to investigate this region.

\section{CONCLUSIONS}

In this paper, we have used the generalized Langevin equation to investigate fluctuations in simple liquids in the framework of a generalized hydrodynamic description. The various transport coefficients appearing in this description have been defined in terms of time correlations of the dynamical variables, and computed numerically whenever possible for argonlike liquids. The results, such as the variation of viscosities with wavelength, may be applicable to other simple liquids. In view of the good quantitative agreement with Rahman's data for all values of $k$ and $\omega$ encountered in neutron scattering, we may expect formula (5.2) to be applicable to the interpretation of coherent neutron scattering from dense fluids. The formalism developed in this paper enables one to calculate correlations between other pairs of
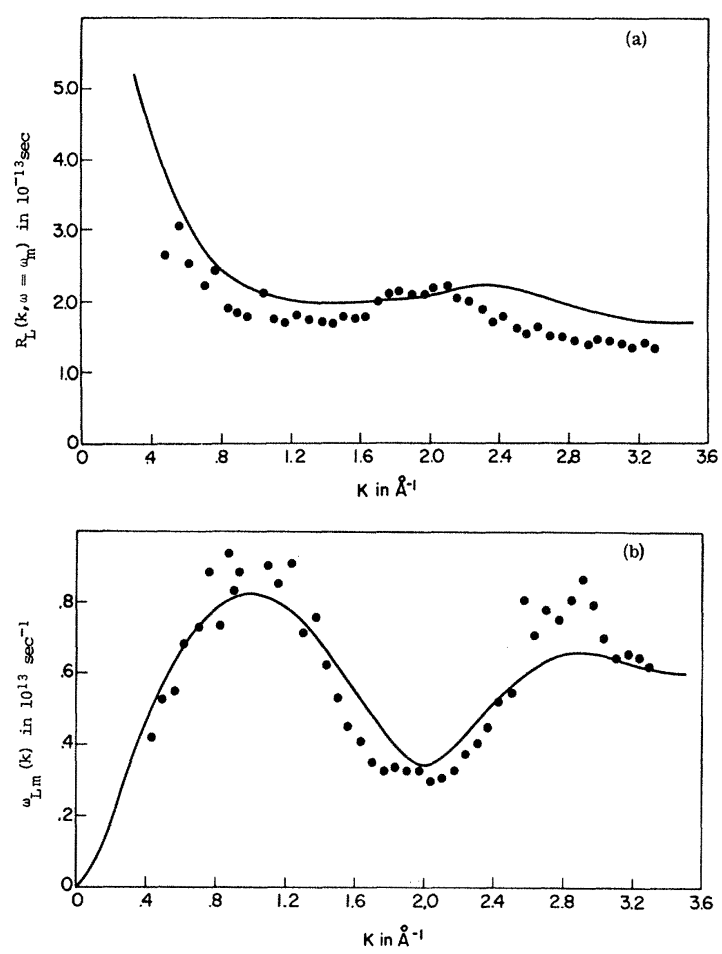

FIG. 7. (a) Maxima of the longitudinal current-current correlation function versus wave number $k$. The points are from Rahman. (b) Frequency at which the longitudinal current-current correlation function is maximum.

hydrodynamic variables, e.g., $\left\langle E(t) E^{*}\right\rangle$ and $\left\langle\overrightarrow{\mathrm{q}}(t) \overrightarrow{\mathrm{q}}^{*}\right\rangle$, etc. Computer results for these correlation functions in conjunction with the analytical calculations will shed light on the $k$ and $\omega$ dependence of the thermal parameters $\gamma_{L}(k), \omega_{3}(k)$, and $D_{L}(k)$ as we have demonstrated for the viscosities.

\section{ACKNOWLEDGMENTS}

We wish to thank Professor R. K. Osborn and Professor J. Duderstadt for their helpful comments and reviewing and editing of this paper. Also, we thank Professor S. Yip for interesting discussions. This work was supported in part by the National Science Foundation.
${ }^{*}$ Permanent address: University of Michigan, Ann Arbor, Mich.

${ }^{1}$ A. Rahman, Phys. Rev. 136, A405 (1964); Phys. Rev. Letters 19,420 (1967).

${ }^{2}$ A. Rahman, Neutron Inelastic Scattering (International Atomic Energy Agency, Vienna, 1968), Vol. I。

${ }^{3}$ M. Nelkin, J. M. J. VanLeeuwen, and S。 Yip, Inelastic Scattering of Neutrons in Solids and Liquids (International
Atomic Energy Agency, Vienna, 1965), Vol。II, pp。 $35-57$.

${ }^{4}$ J. M. J. VanLeeuwen and S. Yip, Phys. Rev. 139, A1138 (1965).

${ }^{5}$ M. Nelkin and S. Yip, Phys. Fluids $\underline{9}, 380$ (1966).

${ }^{6}$ J. J. Greytak and G. B. Benedek, Phys. Rev. Letters 17,179 (1966).

${ }^{7} \mathrm{M}$. Nelkin and S. Ranganathan, Phys. Rev. 164, 222 
(1967); S. Ranganathan and M. Nelkin, J. Chem. Phys. 47, 4056 (1967).

${ }^{8} \mathrm{M}$. Nelkin and P. J. Ortoleva, IAEA Symposium Inelastic Neutron Scattering (International Atomic Energy Agency, Copenhagen, 1969).

${ }^{9}$ H. B. Callen and T. A. Welton, Phys. Rev. 83,34 (1951); R. Kubo, Tokyo Summer Lectures in Theoretical Physics, Part 1, Many-Body Theory, 1966 (unpublished).

${ }^{10}$ L. D. Landaue and E. M. Litschitz, Statistical Physics (Pergamon, London, 1959).

${ }^{11}$ S. M. Rytov, Zh. Eksperim。i Teor。Fiz. $\underline{33}, 166$ (1957) [Soviet Phys。JETP 6 , 130 (1958)]。

${ }^{12}$ L. P. Kadonoff and P. C. Martin, Ann. Phys. (N.Y.) $\underline{24}, 419$ (1963).

${ }^{13}$ R. Mountain, Rev。 Mod. Phys。38, 205 (1966)。

${ }^{14} \mathrm{C}$. H. Chung and S. Yip, Phys. Rev。182, 323 (1969)。

${ }^{15} \mathrm{R}$. Zwanzig, in Lectures in Theoretical Physics, edited by W. E. Brittin, W. B. Downs, and J. Downs (Interscience, New York, 1961), Vol. III.

${ }^{16}$ H. Mori, Progr. Theoret. Phys. (Kyoto) 33, 423 (1965).

${ }^{17}$ H. Mori, Progr. Theoret. Phys. 34, 399 (1965).

${ }^{18}$ A. Z. Akcasu and J. J. Duderstadt, Phys. Rev. 188, 479 (1969).

${ }^{19} \mathrm{R}$. Zwanzig, Phys。Rev。144, 170 (1966).

${ }^{20}$ M. C. Wang and G. E. Uhlenbeck, Rev。Mod. Phys。 17,323 (1965); S。Chandrasekhar, ibid。15, 1 (1943)。

${ }^{21}$ P. Schofield, Proc. Phys.Soc。(London) 88,149 (1966).

${ }^{22} \eta_{T}(k, i \omega)$ will be identified later as the shear viscosity

[cf. (4.54)] in an approximate sense.

${ }^{23}$ R. Zwanzig and R. Mountain, J. Chem. Phys. $\underline{43}$, 4464 (1965).

\title{
Time-Correlation Functions, Memory Functions, and Molecular Dynamics*
}

\author{
G. D. $\operatorname{Harp}^{\dagger}$ \\ Chemistry Department, Brookhaven National Laboratory, Upton, New York 11973 \\ and \\ Chemistry Department, Columbia University, New York, New York 10027 \\ and \\ B. J. Berne \\ Chemistry Department, Columbia University, New York, New York 10027 \\ (Received 29 January 1970)
}

\begin{abstract}
The memory functions for the velocity, angular-momentum, and dipolar autocorrelation functions from a series of molecular-dynamics studies of liquid carbon monoxide are examined. The velocity and angular-momentum memory functions decay initially almost to zero in a Gaussian fashion. However, their long-time behavior has a much slower time dependence. The dipolar memory function from a simulation using a strong noncentral potential is approximately this system's angular-momentum autocorrelation function. Approximate velocity and angular-momentum correlation functions are generated from approximate memory functions and the results are compared to experiment. Gaussian memories based on the second and fourth moments of the corresponding autocorrelation functions give the best agreement with experiment. However, none of the approximate memories examined adequately represents the long-time behavior of the experimental memories. The static atomic radial distribution functions are given and are shown to depend upon the strength of the orientational parts of the pair potential used in the dynamics calculations. The non-Gaussian characteristics of the Van Hove self-correlation functions are examined and shown to depend on the potential and number of particles used in the dynamics calculations. The intermediate scattering function and its memory are also examined.
\end{abstract}

\section{INTRODUCTION}

A number of experimental methods exist for probing the structure and molecular dynamics of liquids. X-ray and neutron-scattering experiments determine the structure factor $S(\vec{K})$ which is related by a Fourier transform to the pair-correlation function of the liquid. Inelastic neutronscattering experiments determine the dynamic form factor $S(\vec{K}, \omega)$, first introduced by Van Hove. ${ }^{1}$ $\mathrm{S}(\overrightarrow{\mathrm{K}}, \omega)$ is related to the transition rate for the liquid system to absorb momentum $\hbar \overrightarrow{\mathrm{K}}$ and energy $\hbar \omega$ from the thermal neutron beam. Moreover, the dynamic form factor $\mathrm{S}(\overrightarrow{\mathrm{K}}, \omega)$ is the Fourier transform of the correlation function of the number densities at two different space-time points. This same function plays an important role in the 